\title{
Sulle varietà algebriche a curve-sezioni di genere tre.
}

\author{
Memoria di Ugo Morin (a Padova).
}

Sunto. - Delle varietà algebriche a 3 dimensioni (non coni o luoghi di $\infty^{1}$ piani) a curve sezioni di genere $\pi=3$ si danno $i$ tipi proiettivamente distinti. Questi, ad ecceione della $\nabla_{3}{ }^{(4)}$ dell' $\mathbf{S}_{4}$, sono razionali. I loro sistemi rappresentativi forniscono tutti $i$ sistemi lineari di superficie dell' $\mathrm{S}_{3}$, cremonianamente distinti, a curva caratteristica di genere 3 , semplici e di grado $>4$.

Lo studio delle superficie algebriche a curve-sezioni di genere tre ha avuto una sistemazione definitiva in classici lavori di CAstelnuovo e Sconza (n. 1). Nella presente Memoria verifico che una varietà algebrica a tre dimensioni $V_{3}^{(n)}$, d'ordine $n$, normale, non cono, a curve-sezioni di genere tre, può essere:

1) una rigata $V_{3}^{(12)}$ dell' $S_{11}$, contenente $\infty^{1}$ superficie di VERoNESE, o una sua proiezione;

2) una rigata $V_{3}^{(13)}$ dell' $S_{12}$, con un piano direttore, contenente $\infty^{2}$ superficie del nono ordine, a curve-sezioni ellittiche; o una sua proiezione;

3) la $V_{3}{ }^{(8)}$ dell' $S_{7}$, sezione completa d'un $S_{1} \cdot$ cono di Veronese con una quadrica generica dell' $S_{7}$, o una sua proiezione;

4) una $V_{3}^{(5)}$ dell' $S_{4}$ con un piano triplo;

כ) una $V_{3}^{(6)}$ dell' $S_{5}$, sezione residua d' un cono quadrico dell' $S_{5}$, luogo di $\infty^{1}$ spazi $S_{3^{\prime}}$, con un'ipersuperficie del quarto ordine, che passa per due di quegli $S_{3}$;

6) una $V_{3}^{(7)}$ dell' $S_{6}$, intersezione residua d'una varietà algebrica $M_{4}^{(3)}$ (del terzo ordine e di dimensione quattro) razionale normale (luogo di $\infty^{1} S_{3}$ ), con un'ipersuperficie cubica che passa per un cono quadrico $K_{3}^{\left({ }^{2}\right)}$ dell' $M_{4}^{(3)}$;

7) una $V_{3}^{(r+1)}$ dell' $S_{r^{n}}(7 \leq r \leq 13)$ intersezione residua di una $M_{4}^{(r-3)}$ (luogo di $\infty^{1} S_{3}$ ), con una quadrica dell' $S_{r}$ che passa per $r-7$ spazi $S_{3}$ dell' $M_{4}^{(r-3)}$;

8) un'ipersuperficie del quarto ordine dell' $S_{4}$ (priva di superficie multiple);

9) il luogo di un sistema algebrico $\infty^{1}$ di piani, di genere tre.

Colpisce la mancanza in questo elenco di varietà a superficie-sezioni d'irregolarita uno, le quali sono risultate tutte dei coni. Ad eccezione degli ultimi 
due tipi, queste varietà sono certamente razionali e forniscono, con la loro rappresentazione nell' $S_{3}$, tutti i tipi cremonianamente distinti di sistemi lineari di superficie a carva-sezione variabile di genere tre, di grado $n>4$ (non rappresentativi di coni). Questi sono (il $\mathrm{n}^{\circ}$. tra parentisi indicando la relativa rappresentazione analitica) sistemi di:

$$
\text { per } n=12-v, \quad(0 \leq v \leq 7),
$$

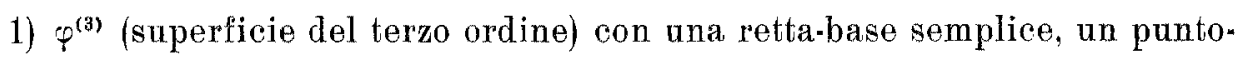
base doppio e $\vee$ punti-base semplici, (1);

$$
\text { per } n=13-v, \quad(0 \leq v \leq 8) \text {, }
$$

2) $\varphi^{(3)}$ con un punto-base doppio biplanare, in esso un piano tangente fisso e $\gamma$ punti-base semplici, (2);

$$
\text { per } n=8-v, \quad(0 \leq v \leq 3) \text {, }
$$

3) $\varphi^{(4)}$ con un tacnodo, quattro rette-base per esso, e una $C^{(t)}$-base, sezione di una $\varphi^{(t)}$ con un piano generico, (3);

oppure un sistema lineare di superficie $\varphi^{(s)}$, a curva-caratteristica iperellittica, con una retta-base $b$ multipla dell' ordine $s-2$, ed inoltre:

$$
\text { per } s=3 \text {, }
$$

4) sulla $b$ due punti-base doppi, eventualmente infinitamente vicini $(n=12),(10)$;

5) tangenti lungo la retta $b(n=11),(24)$;

$$
\text { per } s=4 \text {, }
$$

6) una $C^{(5)}$-base, sezione residua di una $\varphi^{(3)}$ e una $\varphi^{(2)}$ per $b(n=9),(6)$;

7) una $O^{(4)}$-base, di prima specie, di cui $b$ è una corda $(n=10)$, (7);

8) una $C^{(3)}$-base sghemba, semplicemente appoggiata alla $b(n=11)$, (8);

9) due rette-base semplici sghembe $(n=12)$, (9);

10) un punto-base triplo, in oui il cono tangente si spezza in un cono quadrico fisso e in un piano variabile per la $b$, generatrice del cono fisso, e $\vee$ generatrici di questo cono come rette-base semplici $(n=14-v, 0 \leq v \leq 3)$, (11);

11) una $C^{(6)}$-base, sezione d' una $\varphi^{(4)}$ con una $\varphi^{(2)}$ per $b(n=8),(13)$;

12) una retta-base semplice $c$ incidente la $b$ (ed eventualmente ad essa infinitamente vicina, ed una $C^{(4)}$-base, sezjone píana di una $\varphi^{(4)}(n=9),(16)$;

13) un punto-base doppio $B$, e una $C^{(4)}$-base, sezione d'una $\varphi^{(3)}$ con an piano generico per $B(n=10)$, (19);

14) un punto tacnodale $B$ sulla $b$, ed una $C^{(4)}$-base, sezione di una $\varphi^{(t)}$ con un piano per $B(n=10),(20)$; 
15) aventi tra di loro un contatto del secondo ordine lungo due piani tangenti fissi per $b(n=10)$, (21);

16) una retta-base semplice $c$ (eventualmente infinitamente vicina a $b$ ), incidente $b$ in un punto triplo $B$, tangenti lango la $b$ e in $B$ due piani tangenti fissi $(n=11),(25),(26),(27)$;

$$
\text { per } s=5 \text {, }
$$

17) una retta-base doppia $c$, incidente la $b$ in un punto quadruplo $B$, con ivi un cono tangente cubico fisso $(n=14)$, (12);

18) una retta-base semplice $c$, incidente $b$ in un punto $B$, con ivi un piano tangente tacnodale $\beta$ (diverso dal piano $b c$ ), ed inoltre una $C^{(7)}$-base, sezione d' una $\varphi^{(5)}$ con una quadrica per $b$, col piano tangente $\beta(n=-8),(14)$;

19) tre piani tangenti fissi lnngo la $b$, ed una $C^{(5)}$ base, sezione piana di nna $\varphi^{(5)}(n=8),(15)$;

20) un punto $B$ di $b$ quadruplo, ed in esso due piani tangenti fissi per la retta $b$, ed una $C^{(7)}$-base, sezione d' una $\varphi^{(5)}$ con un cono quadrico di rertice $B$ e con la generatrice $b(n=9),(17)$;

21) due piani tangenti fissi lango $b$, ed in un suo punto quadruplo $B$ fisso anche un terzo piano tangente per $b$ e una $C^{(5)}$ base, sezione d' una $\varphi^{(5)}$ con un piano generico per $B(n=9),(18)$;

22) una retta-base semplice $c$, incidente $b$ in un punto quadruplo $B$, con ivi due piani tangenti fissi per $b$, ed inoltre una $C^{(5)}$ base, sezione d' una $\varphi^{(5)}$ con un piano generico per $B(n=10),(22),(23)$;

$23)$ in un suo punto quadruplo $B$ fissi tre piani tangenti per $b$, e una $C^{(5)}$.base, sezione d' una $\varphi^{(5)}$ con un piano generico per $B(n=11)$, (28);

24) tre piani tangenti fissi lungo $b$, e lungo due di essi un contatto del secondo ordine, e in un punto quadruplo $B$ della $b$ fissi due piani tangenti per $b(n=11)$, (29);

25) coi piani tangenti fissi nei punti della $b$, una retta-base semplice $c$, incidente la $b$ in un punto quintuplo $B$, in cui tre piani tangenti per $b$ sono fissi $(n=12),(31)$;

26) con tre piani tangenti fissi lungo la $b$, lungo uno di essi un contatto del secondo ordine, e in un punto qnadruplo della $b$ fissi tre piani tangenti per $b(n=12),(32)$;

27) un punto-base doppio $B$, e una $C^{(8)}$-base, sezione di una $\varphi^{(4)}$, con la retta doppia $b$ e il punto doppio $B$, e d'una $\varphi^{(3)}$, con la retta roppia $b$ e il punto semplice $B(n=8),(33)$;

28) an punto-base doppio $B$ e una $C^{(9)}$-base, sezione d' una $\varphi^{(\hat{)})}$ con una $\varphi^{(3)}$, con la retta-base doppia $b$ e il punto-base semplice $B(n=7)$, (37); 
29) un piano tangente tacnodale $\beta$ in un punto $B$ di $b$, e una $C^{(9)}$-base, sezione d'ana $\varphi^{(5)}$ con una $\varphi^{(3)}$ con la retta doppia $b$ e il piano tangente $\beta$ $(n=7),(38)$;

30) due piani tangenti fissi per $b$ e una $C^{(7)}$.base, sezione d' una $\varphi^{(5)}$ con una $\varphi^{(2)}$ per $b(n=7),(39)$;

$$
\text { per } s=6,
$$

31) tre rette-basi $c, d$, $e$ rispettivamente complanari con $b$ e ad essa infinitamente vicine, di cui $c$ doppia e lungo le rette semplici $d, e$ un contatto del secondo ordine, ed inoltre in un punto quintuplo della $b$ fissi due piani tangenti, oltre il piano $(b c),(n=12),(30)$;

32) un punto doppio $B$, ed una $C^{(12)}-$ base, sezione $d$ ' una $\varphi^{(5)}$ con la retta tripla $b$ e il punto doppio $B$, con una $\psi^{(5)}$, con la retta quadrupla $b$ e il punto semplice $B$, le quali hanno inoltre in comune una generatrice della rigata $\left.\psi^{(5)}\right)$, $(n=6),(43)$;

33) un punto doppio $B$, ed una $C^{(12)}$-base, sezione d' una $\psi^{(5)}$, colla retta quadrupla $b$ e il punto semplice $B$. con una $\varphi^{( \pm)}$, colla retta doppia $b$ e il punto doppio $B(n=6)$, (44);

34) una $C^{(13)}$ base, sezione di due superficie del quinto ordine, colla retta $b$ rispettivamente tripla e quadrupla, tangenti in un punto $B$, il quale è doppio per le $\varphi^{(6)},(n=5),(45)$.

\section{Nozioni introduttive.}

1. Una superficie algebrica irriducibile $F_{2}^{(n)}$, d'ordine $n$, dello spazio lineare $S_{r-1}(r-1 \geq 3)$, a curve-sezioni $C$ cogli iperpiani dell' $S_{r-1}$, di genere $\pi=3$, può essere $\left({ }^{1}\right),\left({ }^{2}\right)$,

I) una superficie d'irregolarità 1 dei seguenti tre tipi:

a) $F_{2}{ }^{(8)}$ dell' $S_{5}$ contenente un fascio ellittico di coniche e un sistema $\infty^{t}$ di cubiche ellittiche, di grado 1 e indice 2 ; o una sua proiezione;

b) $F_{2}{ }^{(8)}$ dell' $S_{5}$ contenente un fascio ellittico di coniche e un sistema algebrico $\infty^{2}$ di quartiche di prima specie, di grado e indice 2 ; o una sua proiezione;

c) $F_{2}^{\left({ }^{(8)}\right.}$ dell' $S_{6}$ contenente una rete lineare di quartiche di prima specie, di grado 2 ;

(1) CASTmunuovo $\mathrm{G}_{*}$, Sulle superficie algebriche le eut sezioni sono curve di genere 3 ("Atti della R. Accademia delle Scienze di Torino», t. XXV, 1890].

(2) SconzA G., Le superficie a cuvve-sezioni di genere 3 ["Annali di Matematica ", t. XVI (1909), pagg. 255-327 e t. XVII (1910), pagg. 281-321]. 
oppure una superficie razionale rappresentabile sul piano

II) da un sistema lineare di quartiche;

III) da un sistema lineare di sestiche con 7 punti-base doppi;

IV) da un sistema lineare di curve iperellittiche;

oppure

V) una superficie del quarto ordine dell' $S_{3}$;

VI) una rigata di genere 3.

Nei successivi quattro capitoli studieremo le varietà algebriche $V_{3}^{(\boldsymbol{m})}$, a tre dimensioni e dell' ordine $n$, normali dello spazio lineare $S_{r}(r \geq 4)$ a curvesezioni $O$ di genere $\pi=3$, a seconda che le relative superficie-sezioni, cogli $S_{r-1}$ dell' $S_{r}$, appartengono ad uno dei primi quattro dei tipi ora elencati.

Invece lo studio delle varietà corrispondenti agli ultimi due tipi, cioè dell' ipersuperficie quartica $V_{3}^{(4)}$ dell' $S_{4}$ o di un insieme algebrico di piani $\infty^{4}$ di genere 3 , non viene approfondito, $\left({ }^{3}\right)$.

\section{Captrolo I.}

\section{$V_{3}^{(n)}$ a superficie-sezioni irregolari.}

2. In questo primo capitolo dimostreremo che una $V_{3}^{(n)}$ dell' $\mathrm{S}_{\mathrm{r}}$, a curvesezioni di genere $\pi=3$ e a superficie-sezioni d'irregolarità $\delta=1$, è un cono.

Cominciamo col supporre che la superficie-sezione $F_{2}^{(n)}$ della $V_{3}^{(n)}$ con un $S_{r-1}$ generico dell' $S_{r}$. sia del tipo I, a) (n. $\left.1,\left(^{4}\right)\right)$. Se è $n \geq 7$, la $F_{2}^{(n)}$ contiene un determinato sistema $\infty^{\prime}$ ellittico di cubiche ellittiche, invece per $n=6$, la $F_{q}^{(6)}$ contiene due.di tali sistemi. Questo sistema (o questa coppia di sistemi) non è contenuto in un sistema di cubiche di dimensione più ampia. Le cubiche sono inoltre unisecanti le coniche del fascio ellittico.

Possiamo supporre che la dimensione $r$ dello spazio d'appartenenza della $V_{3}^{(n)} \operatorname{sia} r=4$ (sostituendo eventualmente alla varietà data una sua generica proiezione). Variando nell' $S_{4}$ l' $S_{3}$ della $F_{2}^{(n)}$, otteniamo nella $V_{3}^{(n)}$ un sistema algebrico $\Sigma$ di $C^{(3)}$ ellittiche, di dimensione $d=4$ (poichè se fosse $d=5$, in una $F_{2}^{(n)}$ generica sarebbero contenute $\infty^{2} C^{(3)}$ ).

(3) Alle $V_{3}^{(4)}$ sono associati interessanti e difficili problemi. Poichè secondo Favo ["Atti R. Accad. di Torino», t. 43 (1907-08), $50(1914-15)]$ la $V_{3}^{(4)}$ senza punti doppi non ò razionale, classificare i particolari tipi di $V_{3}^{(4)}$ razionali! La $V_{3}^{(4)}$ generale è forse unirazionale, come la $V_{r+1}^{(t)}$ dell' $S_{r}$, per $r \geq 7 ?$ [cfr. MonIN U., Sull'unirazionalitò dell'ipersuperficie algebrica del quarto ordine, * Rend. R. Accad. dei Lincei, t. 24 (6) (1986)].

(4) Soonza G., loco cit. (2). 
Sembrano possibili i seguenti due casi :

1) per due punti generici della $V_{3}^{(r)}$ passa un numero finito di $C^{(3)}$ di $\Sigma$;

2) per due punti generici della $V_{3}^{(n)}$ non passa alcuna $C^{(3)}$ di $\Sigma$; e quindi se per due punti particolari passa una $C^{(3)}$ di $\Sigma$, di conseguenza ne passano infinite.

Proviamo che il primo caso effettivamente non può verificarsi. Considerata infatti una conica generica $C^{(2)}$ di una $F_{\mathrm{g}}^{(n)}$ generica, vi sarebbero (almeno) $\infty^{2} C^{(3)}$ di $\Sigma$ che si appoggiano alle coppie di punti della conica $C^{(2)}$. Non è possibile che $\infty^{1}$ di queste $\infty^{2} C^{(3)}$ stiano in una generica $F_{2}^{(n)}$ per la $C^{(2)}$, poichè le $C^{(3)}$ e le coniche dell' $F_{2}^{(n)}$ (delle qủali $C^{(2)}$ è una generica) si tagliano mutuamente in punti, e non in coppie di punti. E neanche è possibile che $\infty^{2} C^{(3)}$ stiano in un medesimo $S_{3}$, poichè allora formerebbero una (o dne) superficie cabiche $\Phi\left({ }^{5}\right)$, e le $\infty^{4} C^{(3)}$ di $\Sigma$ distribuendosi in superficie eubiche $\Phi$, per due punti generici della $V_{3}^{(m)}$ non passerebbe una $C^{(3)}$ di $\Sigma$.

Nel secondo easo, alle $\infty^{2}$ coppie di punti di una $\bar{C}^{(3)}$ di $\Sigma$, si appoggiano $\infty^{3}$ eubiche $C^{(3)}$ di questo sistema. Poichè $\infty^{2}$ di queste $\infty^{3} C^{(3)}$ non possono stare in una-generica $F_{2}^{(n)}$ per la $\bar{C}^{(3)}, \infty^{3} C^{(3)}$ staranno in un medesimo $S_{3}$ per la $\bar{C}^{(3)}$, cioè formeranno una (o due) superficie cubica $\Phi$. Le $\infty^{4} O^{(3)}$ di $\Sigma$ si distribuiscono dunque in $\infty^{1}$ superficie cubiche $\Phi$ (poichè per una $C^{(3)}$ non possono passare $\left.\infty^{1} \Phi\right)$.

Considerato un insieme $\infty^{1}$ irriducibile $\Sigma_{1}$ di superficie $\Phi$, poichè un $S_{3}$ generico sega due $\Phi$ di $\Sigma_{1}$ in due $C^{(3)}$ con un (solo) punto comune, due generiche $\Phi$ di $\Sigma_{1}$ hanno in comune una retta variabile. Le $\Phi$ sono quindi coni con lo stesso vertice $W$, cioè la $V_{3}^{(n)}$ stessa è un cono di vertice $W$.

Nel cono $V_{3}^{(n)}$, le eui generatrici proiettano da $W$ una superficie $F_{2}{ }^{n}$, $(I, a)$, è quindi contenuto un fascio ellittico di coni quadrici, di vertice $W$.

3. Se la superficie-sezione della $V_{3}{ }^{(n)}$ è del tipo $I, b$ (n. 1), quindi $n=8$ (poichè la proiezione da un suo punto di una $F_{2}^{(8)}$ di tipo $\mathrm{I}$, b contiene un sistema $\infty^{\prime}$ di $C^{(3)}$ ellittiche, proiezioni delle $C^{(4)}$ ellittiche che passano per il centro di proiezione), possiamo supporre $r \geq 6$.

Infatti, la deficenza $\delta_{2}$ del sistema caratteristico del sistema completo

(5) I'ordine della superficie $\Phi$ dell' $S_{3}$ non può essere superiore a 3 , poichè contenéndo essa $\infty^{2} C^{(3)}$ ellittiche, queste (nel caso che l'ordine di $\Phi$ fosse naggiore di 3) dovrebbero appartenere a curve-sezioni piane della $\Phi$ spezzate in una $C^{(3)}$ e in una retta; cioè (teorerua di Darboux) la $\Phi$ dovrebbe essere una rigata del quarto ordine con la conica direttrice $C^{(2)}$ (semplice, poichè una rigata con conica doppia ha l'ordine superiore a 4), cioè la $\Phi$ sarebbe razionale; ciò che è assurdo poichè le $C^{(3)}$ sono ellittiche. 
$\left(F_{z}{ }^{(8)}\right),\left(^{6}\right)$, non può superare l'irregolarità superficiale $q_{2}=1$ della $V_{3}^{(8)}$, cioè la deficienza della serie lineare delle sezioni iperpiane della curva-sezione $C^{(8)}$ è al più 1 , dunque $r-2 \geq 8-3-1$, cioè $r \geq 6$.

Da un suo punto generico $A$ la $V_{3}^{(8)}$ si proietta in una $V_{3}^{(7)}$ di un $S_{r_{-1}}$, $(r-1 \geq 5)$, del tipo del numero precedente, sulla quale vi sono $\infty^{1}$ coni quadrici col comune vertice $W$. Poichè questi coni quadrici segano sulle super. ficie-sezioni le coniche del fascio ellittico, essi sono proiezioni di coni quadrici della $V_{3}^{\left({ }^{(8)}\right.}$ stessa; e poichè questi coni quadrici si proiettano in coni con lo stesso vertice $W$, il centro di proiezione $A$ essendo generico, anche i coni quadrici della $V_{3}^{(8)}$ hanno in comune il vertice; cioè la $V_{3}^{(8)}$ stessa è un cono.

4. La superficie-sezione $F_{2}^{(n)}$ della $V_{3}^{(n)}$ sia del tipo I, c, cioè contenga una rete lineare di $C^{(4)}$ di prima specie, di grado 2 , (7).

Supponiamo ancora che la $V_{3}^{(n)}$ appartenga all' $S_{4}$. Sia $C$ la curva-sezione della $V_{3}^{(m)}$ con un piano generico $\propto$ dell' $S_{4}$. Per due punti generici, $P_{1}$ e $P_{2}$, della $C$, passa, dentro alla superficie-sezione $F_{2}^{(n)}$ della $V_{3}^{(n)}$ con un $S_{3}$ generico per $\alpha$, una determinata $C^{(4)}$ della relativa rete di curve ellittiche. Variando $l^{\prime} S_{3}$ per $\alpha$, la $C^{(4)}$ deserive una superficie $\Phi$. Proviamo che la $\Phi$ è del quarto ordine.

Infatti i punti $P_{3}$ e $P_{4}$, ulteriori intersezioni della $C^{(4)}$ col piano $\alpha$ sono fissi al variare della $C^{(4)}$, poichè nel caso contrario un particolare $P_{3}$ potrebbe essere allineato con $P_{1}, P_{2}$, cioè la ręlativa $C^{(4)}$ di $1^{\text {a }}$ specie dovrebbe conte. nere i tre punti allinéati $P_{1}, P_{2}, P_{3}$, senza contenere la loro retta congiungente, ciò che è assurdo. Dunque il piano $\alpha$ ha in comune con la $\Phi$ soltanto i quattro punti (fissi) $P_{1}, P_{2}, P_{3}, P_{4}$ (non potendo inoltre una particolare $C^{(t)}$ contenere come parte la $C$ di $\alpha$, la quale è di ordine superiore a 4), cioè la $\Phi$ è del quarto ordine.

Alle $\infty^{2}$ coppie di punti $P_{1}, P_{z}$ della $C$ di $\alpha$, corrispondono $\infty^{2}$ superficie $\Phi$, le quali segano dunque su una superficie-sezione generica della $V_{3}^{(n)}$ un sistema $\infty^{2}$ di $C^{(4)}$ ellittiche, cioè le curve della rete di quartiche ellittiche ivi esistenti, $\left({ }^{8}\right)$. Poichè il grado di questa rete è due, due $\Phi$ si tagliano in due rette $o$ in una conica variabili. Se due $\Phi$ si tagliano in due rette, la $\Phi$ generica è una rigata (a curve-sezioni $C^{(4)}$ ellittiche e appartenente all' $S$,), cioè un cono. Questi $\infty^{2}$ coni $\Phi$, avendo a due a due in comune una coppia di

(6) Severi F., Fondamenti per la geometria sulle varietà algebriche ["Rendiconti de] Circolo Matematico di Palermo ", t. XXVIII (1909)], teorema VIII.

(7) Scorza G., loo. cit. (2).

(8) Scorza G., loc. cit. (2). 
generatrici, hanno in comune il vertice $W$; cioè la $V_{3}^{(n)}$ stessa è un cono di vertice $W$.

Constatiamo che due $\Phi$ non possono tagliarsi in una conica irriducibile $C^{(2)}$. Infatti, se ciò fosse, la rete $\Phi$ sarebbe composta con una congruenza $K$ di coniche. Poichè i piani di queste coniche sono segati da un $S_{3}$ generico nelle rette di una stella $(\%)$, questi $\infty^{2}$ piani dell' $S_{4}$ passano per una medesima retta s. Ma allora le $\infty^{1}$ coniche di una $\Phi$ (superficie di SEgRE) hanno in comune con questa retta $s$ i medesimi due punti $M, N$; cioè tutte le $\infty^{2}$ coniche della congruenza $K$ (di indice 1) passerebbero per $M$ e $N$. La $V_{3}^{(n)}$ sarebbe allora razionale, in contrasto con l'ipotesi che la sua irregolarità superficiale $q_{2}=1$.

\section{Capitolo II.}

\section{$V_{3}^{(n)}$ la cui superficie-sezione è rappresentabile nel piano da un sistema lineare di quartiche.}

5 Poichè, nell' ipotesi di questo capitolo, la superficie-sezione $F$ della $V_{3}^{(n)}$ è razionale, lo è anche la $V_{3}{ }^{(n)},\left({ }^{40}\right)$. Quindi se la $V_{3}^{(n)}$ è normale, tali saranno anche le curve-sezioni (di genere $\pi=3$ ), cioè, $r$ essendo la dimensione dello spazio. d'appartenenza della $V_{3}^{(n)}$, sarà $n=r+1$. Le curve $C^{\prime}$, agginnte alle curve-sezioni $O$ della $F$ formano una rete omaloidica di quartiche razionali. Il sistema $\Sigma=\left(F+F^{\prime}\right)$ sega (oltre ad eventuali componenti fisse) sulla $F$ la serie completa $\left|C^{\prime}\right|,\left(^{11}\right)$; cioè $\Sigma$ è composto con una congruenza di rette di indice 1. La $V_{3}^{(n)}$ è dunque in questo caso rigata, e le superficie di $\Sigma$, soppresse eventnali componenti fisse, sono rigate del quarto ordine (e la dimensione di s̀ è 2 ).

Si constata direttamente che $V_{3}^{(n)}$ del tipo ora considerato sono:

I) la rigata $V_{3}^{(12)}$ dell' $S_{41}$, le cuj generatrici conginngono le coppie di punti corrispondenti di dne superfieie di VERONESE (di due $S_{5}$ complementari dell' $\left.S_{14}\right)$, in una corrispondenza birazionale tra queste due stiperficie nella quale si corrispondono le rispettive reti di coniche;

II) la rigata $V_{3}^{(13)}$ dell' $S_{12}$, le cui generatrici congiungono le coppie di punti corrispondenti di un piano $\alpha$ e d'una superficie $F_{2}^{\left({ }^{(9)}\right.}$, di un $S_{9}$ comple-

(9) Castelnuovo G., loc. cit. (1).

(10) FANO G., Sulle varietà algebriche a tre dimensioni a sinperfieie-sezioni razionali ["Annali di Mat.", t. 24 (3), (1915)], pagg. 49.88. - Morin U., Sulla classificazione proiet* tiva delle varietò a superficie-sezioni razionali ["Annali di Mat. ", t. 18 (4), (1939)].

(11) Severi F, loc. oit. $\left({ }^{6}\right)$. 
mentare di $x$ e a curve-sezioni ellittiche, in una corrispondenza birazionale che alla rete delle rette del piano fa corrispondere la rete delle cubiche del. $\mathrm{l}^{\prime} \mathrm{F}_{2}{ }^{(9)}$.

Infatti, la $V_{3}$ del tipo I ha effettivamente l'ordine 12, poichè un $S_{10}$ generico per la prima superficie di Veronese taglia la seconda in una $C^{(4)}$, cui corrisponde (nella corrispondenza birazionale tra le due superficie di VkRo. NESE) una $C^{(4)}$ sulla prima, cioè le generatrici della $V_{3}$ contenente nell' $S_{10}$ formano una rigata dell'ottavo ordine, quindi la superficie-sezione dell' $S_{10}$ colla $V_{3}$ ha l'ordine $4+8=12$. La $V_{3}^{(12)}$ essendo normale nell' $S_{11}$ (il suo spazio d'appartenenza non potendo superare quello delle due superfieie di VERoNese), il genere delle sue curve-sezioni (normali) risulta $\pi=12-9=3$. Analoga verifica si può fare per la $V_{3}$ del tipo II, segandola con un $S_{11}$ per l' $S_{9}$ dell' $F_{2}{ }^{(9)}$.

Il sistema $\Sigma$ delle $\infty^{2}$ rigate $R_{2}{ }^{(4)}$ è dato, nel primo caso dalle $R_{z}{ }^{(4)}$ che segano le due superficie di VERONESE in coniche, nel secondo caso dalle $R_{2}^{(t)}$ che segano il piano $\alpha$ in rette (e quindi la $F_{2}^{(9)}$ in cubiche).

Nei successivi numeri 6 e 7 proveremo che qualunque $\mathrm{V}_{3}{ }^{(\mathrm{r}+1)}$ dell' $\mathrm{S}_{\mathrm{r}}$, le cui superficie-sezioni sono del tipo considerato in questo capitolo, coincidono con la $\mathrm{V}_{3}{ }^{(2)}$ o la $\mathrm{V}_{3}{ }^{(13)}$ ora descritte, oppure con una loro proiezione.

6. La generica rigata $R_{2}^{(4)}$ di $\Sigma$ contenga un fascio lineare di coniche $C^{(2)},\left(^{(2)}\right.$. Le $\infty^{1} C^{(2)}$ per un generico punto $P$ della $V_{s}^{(2+1)}$, appartenenti alle $\infty^{1} R_{2}^{(4)}$ per $P$ (le quali hanno in comune una generatrice $p$ e formano un fascio $\Sigma_{p}$ ), generano una superficie $\Phi$.

Le $R_{2}^{\left({ }^{(4)}\right.}$ della rete $\Sigma$ segnano sulla $\Phi$ una rete omaloidica di curve $|\boldsymbol{\Gamma}|$. Infatti due generiche $R_{2}{ }^{(4)}$ hanno come unico elemento (variabile) comune una generatrice $g$. Questa $g$ appartiene ad una determinata $R_{2}{ }^{(4)}$ del fascio $\Sigma_{p}$, quindi segna sulla $\Phi$ un punto (sezione completa di due curve della rete $|\Gamma|$. Ma la rete $|C|$ contiene totalmente $\left({ }^{13}\right)$ le $\mathrm{C}^{(2)}$ segate dal fascio $\Sigma_{p}$, cioè si tratta di una rete di coniche. Dunque la $\Phi$ è una superficie di Vkroness, o una sua proiezione.

Siano. $\Phi$ e $\Psi$ due superficie del tipo ora considerato. Se il loro spazio d'apparteñenza è l' $S_{11}$, la $V_{3}^{(\hat{r}+1)}$ è la $V_{3}^{(12)}$ del tipo I descritto. Se il loro

(12) Le direttrici di ordine minimo di una $R_{2}^{\left({ }^{(1)}\right)}$ sono $\infty^{1}$ coniche oppure, in particolare, una retta [efr. Conforto F., Le superficie razionali (Nicola Zanichelli, Bologna 1939), pagg. 103 e 119].

(13) Poichè il punto generico $P$ della $V_{3}{ }^{(r+1)}$ non è multiplo per alcuna $R_{2}^{(4)}$, le $\infty^{1} C^{(2)}$ per $P$ sono irriducibili, cioè la retta $p$ non fa parte della superficie $\Phi$. Quindi la completa intersezione di una $R_{2}{ }^{(4)}$ per $P$ con la $\Phi$ è una $C^{(2)}$. 
spazio d'appartenenza ha la dimensione $r=11-(v+1),(0 \leq v-\leq 6)$, immer. giamo l' $S_{v}$. in un $S_{1 !}$.

La $\Phi$ appartenga ad un $S_{5-\lambda}(0 \leq \lambda \leq 3)$ e la $\Psi$ ad una $S_{5-\mu}(0 \leq \mu \leq 3)$, i quali abbiano in comune uno spazio di dimensione $\sigma$; di modo che $r=10-$ $-(\lambda+\mu+\sigma)$, cioè $v=\lambda+\mu+\sigma$. Scegliamo nell' $S_{11}$ uno spazio $S_{v}$, comple. mentare dell' $S_{r}$, $\theta$ indichiamo con $S_{\lambda^{\prime}}$ e $S_{\mu^{\prime}}$ gli spazi che dall' $S_{v}$ proiettano $S_{5-\lambda}$ e $S_{5-\mu}$. Sarà quindi $\lambda^{\prime}=\nu+5-\lambda+1=6+\sigma+\mu, \mu^{\prime}=\nu+5-\mu+$ $+1=6+\sigma+\lambda$. Fissati nell' $S_{3}$ due spazi indipendenti $S_{\lambda-1}$ e $S_{\mu-1}(\lambda-1+$ $+\mu-1+1=\lambda+\mu-1 \leq \lambda+\mu+\sigma=v)$, scegliamo nell' $S_{\lambda^{\prime}}$ un $S_{5}$ generico per l' $S_{\lambda-1}$ e nell' $S_{k^{\prime}}$ un $S_{5}$ generico per l' $S_{\mu-1}$. È quindi possibile scegliere nell' $S_{5}$ dell' $S_{2,}$ una superficie di VERONESE $\Phi$ ', la cai proiezione dall' $S_{y}$ sull' $S_{2}$. sia la $\Phi$; ed analogamente è possibile scegliere nell' $S_{5}$ dell' $S_{\mu^{\prime}}$ una su-

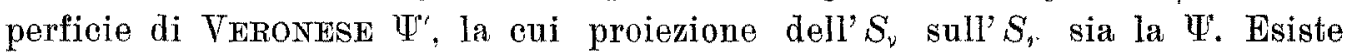
allora una rigata $V_{3}^{(12)}$, appoggiata alla superficie di VkRONEsE $\Phi^{\prime}$ e $\Psi^{\prime}$, la cui proiezione dall' $S_{y}$ sull' $S_{r}$, e la $V_{3}^{(r+1)}$ considerata.

Per trovare della $V_{3}^{(12)}$ dell' $S_{11}$ il sistema lineare rappresentativo nell' $S_{3}{ }^{*}$, osserviamo che la $V_{3}^{\left({ }^{(2)}\right.}$ si proietta biunirocamente dall' $S_{5}$ di una sua $R_{3}{ }^{(4)}$ in una $V_{3}{ }^{(3)}$ di un $S_{5}$ complementare. La $V_{3}{ }^{(3)}$ è rappresentata nell' $S_{3}{ }^{*}$ dal sistema delle quadriche $\varphi^{(2)}$ con una retta-base semplice $b$ ed un punto-base semplice $B$ (non appartenente alla $b$ ). Alle $R_{2}^{(4)}$ della $V_{3}^{(12)}$ corrispondono le $\infty^{2}$ quadriche della $V_{3}^{(3)}$, ed infine gli $\infty^{2}$ piani dell' $S_{3}{ }^{*}$ per il punto $B$.

Poichè nella $V_{3}^{(12)}$ il sistema delle superficie-sezioni $|F|$ è la somma del sistema lineare $\Sigma$ delle $R_{z}^{(4)}$ e del sistema delle sezioni residue della $V_{3}^{(12)}$ cogli $S_{80}$ per una $V_{3}^{(12)}$, si può ottenere il sistema lineare rappresentativo della $V_{3}^{\left({ }^{(2)}\right)}$ nell' $S_{3}^{*}$, sommando il sistema di $\varphi^{(2)}$ rappresentativo della $V_{3}^{(3)}$ del$l^{\prime} S_{5}$ col sistema dei piani pel punto $B$. Si ottiene così come sistema lineare rappresentativo nell' $S_{3}^{*}$ della $V_{3}{ }^{(12)}$ dell' $S_{11}$, il sistema lineare delle superficie cubiche $\varphi^{(3)}$ con una retta-base semplice b ed un punto-base doppio $\mathrm{B}$, non ap. partenente alla b.

Per dare la rappresentazione analitica nell' $S_{3}(x, y, z)$ affine di questo sistema lineare di superficie faremo (qui e nel seguito) le convenzioni di in* dicare con $\lambda_{0}, \lambda_{1}, \lambda_{2}, \ldots ; \bar{\lambda}_{0}, \bar{\lambda}_{1}, \bar{\lambda}_{2}, \ldots$ coefficienti numerici rispettivamente variabili o costanti, e con $F_{i}, G_{i}, H_{i}, \ldots ; \bar{F}_{i}, \bar{G}_{i}, \vec{H}_{i}, \ldots$ polinomi nella sola variabile $\approx$ a coefficienti rispettivamente variabili o costanti, di grado uguale al rispettivo indice $i$. Scelta in particolare come $b$ la retta impropria del piano $(x y)$ e come punto $B$ l'origine, l'equazione del nostro sistema lineare di $\varphi^{(3)}$ è

$$
F_{1} x^{2}+G_{1} x y+H_{1} y^{2}+\left(L_{1} x+M_{1} y+N_{1} z\right) z=0
$$


7. Supponiamo ora che la generica rigata $R_{2}^{(4)}$ del sistema $\Sigma$ (n. 5) contenga una direttrice rettilinea $l$. Sulla $R_{2}{ }^{(4)}$ è individuato un sistema lineare $\infty^{3}$ di cubiche gobbe $C^{(3)}$, di grado 2 .

Fissiamo due generiche superficie di $\Sigma, G$ e $H$, e su ciascuna di esse una cubica gobba generica, rispettivamente $C_{g}{ }^{(3)}$ e $C_{h}{ }^{(3)}$. Indichiamo con $\Sigma_{P}$ il fascio lineare di superficie di $\Sigma$ che passano per un punto $P$, cioè per una genera. trice $p$, della $V_{3}^{(r+1)}$. Una $R_{2}{ }^{(4)}$ di $\Sigma_{P}$ ha in comune colla rigata $G$ una generatrice $g$ e quindi ha in comune colla cubiea $C_{g}{ }^{(3)}$ un solo punto $P_{g}$ (comune a $C_{g}{ }^{(3)}$ e $g$ ). Analogamente l' $R_{2}^{\left({ }^{(t)}\right.}$ ha in comune colla $C_{h}^{(3)}$ della rigata $H$ un solo punto $P_{h}$. Per i tre punti $P, P_{g}, P_{h}$ dell' $R_{z}^{(4)}$ passa una determinata cubica gobba $C^{(3)}$. Al variare della $R_{2}^{(4)}$ nel fascio $\Sigma_{P}$, le $\infty^{1} C^{(3)}$ descrivono una superficie $\Phi$. Due $C^{(3)}$ di $\Phi$ hanno in comune il solo punto $P$.

Poichè due $R_{z}{ }^{(4)}$ generiche di $\mathbf{S}$ hanno in comune una sola generatrice (variabile), $\Sigma$ segna su $\Phi$ una rete omaloidica (n. 6 ) che contiene totalmente $\left({ }^{14}\right)$ di fascio di cubiche gobbe per $P$, cioè la superficie $\Phi$ è rappresentabile su un piano da un sistema lineare di cubiche.

Poichè un punto generico della $\Phi$, interpretato alla stregua del punto $P$, individua la stessa superficie $\Phi$ (il fascio di $C^{(3)}$ per il nuovo punto $P$ appartenendo alla rete di $C^{(R)}$ della $\left.\Phi\right)$, la retta $p$ per $P$ non appartiene alla $\Phi$ (poichè la $\Phi$ non può essere composta con generatrici della $V_{3}^{(r+1)}$ ). Ciò significa che la direttrice $l$ di nessuna delle $\infty^{1} R_{3}{ }^{(4)}$ di $\Sigma_{P}$ passa per $P$, cioè che le $\infty^{2}$ rette $l$ delle $R_{z}^{(4)}$ della rete $\Sigma$ (non invadendo tutta la $V_{3}^{\left(r^{2}+1\right)}$ ) o generano un piano $\alpha$ o coincidono in una retta fissa $\alpha$. Trattiamo separatamente i due casi.

8. Se le rette $l$ generano un piano, le generatrici della $V_{3}^{\left({ }^{2}+1\right)}$ generano tra la superficie $\Phi$ e questo piano una corrispondenza birazionale, nella quale alla rete delle $C^{(3)}$ della $\Phi$ corrisponde la rete delle rette $l$ del piano $\alpha$; e quindi viceversa alle curve-sezioni della $\Phi$ corrispondono cubiche del piano $\alpha$. Da ciò segue direttamente che la $\Phi$ è a curve-sezioni ellittiche. Infatti se $\Phi$ fosse a curve-sezioni razionali, essa sarebbe una rigata alle cui generatrici corrisponderebbero le rette di un piano del piano $\alpha$. Queste coppie di rette corrispondenti individuerebbero un fascio razionale di quadriche della $V_{3}^{(r+1)}$, cioè essa sarebbe (n. 1) a curve-sezioni iperellittiche, in contrasto coll'ipotesi fatta in questo capitolo.

(14) Poichè il punto generico $P$ della $V_{3}^{(r+1)}$ è semplice per ciascuna $R_{2}^{(4)}$ di $\Sigma_{P}$, nes* suna delle o $\sigma^{1} G^{(3)}$ per $P$ contiene come parte la retta $p$. Dunque una $R_{2}^{(4)}$ di $\Sigma_{P}$ ha in co* mune colla $\Phi$ (oltre ad eventuali elementi base di $\Sigma$ ) soltanto la $C^{(3)}$. 
Se lo spazio d'appartenenza della superficie $\Phi$ e del piano $x$ è un $S_{12}$, la $V_{3}^{\left({ }^{(13)}\right.}$ è del tipo II già descritto (n. 5). Se il loro spazio d'appartenenza ha la dimensione $r=12-(v+1),(0 \leq v \leq 7)$, immergiamo l' $S$, in un $S_{11}$.

Lo spazio $S_{9-\lambda}(0 \leq \lambda \leq 6)$ d'appartenenza della $\Phi$ e il piano $\alpha$ abbiano in comune uno spazio di dimensione $\sigma(-1 \leq \sigma \leq 2)$, di modo che sarà $r=$ $=(9-\lambda)+2-\sigma=12-(\lambda+\sigma+1)$, cioè $v=\lambda+\sigma$. Soegliamo nell' $S_{12}$ uno spazio $S_{\nu}$, complementare dell' $S_{r}$, e indichiamo con $S_{\mu^{\prime}}$ e $S_{\lambda^{\prime}}$ gli spazi che dall' $S_{y}$ proiettano, il piano $\approx$ e l' $S_{9-\lambda}$. Sarà quindi $\lambda^{\prime}=(9-\lambda)+(\lambda+\sigma)+$ $+1=10+\sigma, \mu^{\prime}=2+(\lambda+\sigma)+1=3+\sigma+\lambda$. Fissato nell' $S_{y}$ uno spazio generico $S_{\lambda_{-1}}$, scegliamo nell' $S_{\lambda^{\prime}}$ un $S_{9}$ generico per l' $S_{\lambda_{-1}}$ e nell' $S_{\mu^{\prime}}$ un piano generico $\alpha^{\prime}$. È quindi possibile scegliere nell' $S_{9}$ dell' $S_{\lambda^{\prime}}$ una superficie $F_{2}{ }^{(9)}$, la cui proiezione dall' $S_{y}$ sull' $S_{y}$. sia la $\Phi$. Esiste allora una rigata $V_{3}{ }^{(13)}$, appoggiata alla $F_{2}^{(9)}$ e al piano $\alpha$, la cui proiezione dall' $S_{2}$ sull' $S_{r}$ è la $V_{3}^{(r+1)}$ considerata.

Per trovare il sistema lineare rappresentativo della $V_{3}^{(13)}$ dell' $S_{12}$ nell' $S_{3}^{*}$, cominciamo con proiettare la $V_{3}^{(13)}$ dall' $S_{5}$ di una $R_{2}^{(4)}$ su un $S_{6}$ complementare. Otteniamo nell' $S_{6}$ un cono $K_{3}$ di Veronese, rappresentato nell' $S_{3}{ }^{*}$ dalle quadriche col piano tangente $\tau$ fisso in un punto $B$.

Alle $R_{2}{ }^{(t)}$ della $V_{3}^{(13)}$ corrispondono su $K$ i coni quadrici (che dal vertice di $K$ proiettano le coniche di una superficie di Veronese), quindi nell' $S_{3}^{*}$ i piani per il punto $B$. Danque (n. 6) la $V_{3}^{(43)}$ dell' $S_{12}$ è rappresentata nell' $S_{3}$ * dal sistema lineare delle superficie $\varphi^{(3)}$ con un punto doppio biplanare $\mathrm{B}$, ed in esso un piano tangente $\tau$ fisso.

Per dare di questo sistema linoare di $\varphi^{(3)}$ la rappresentazione analitica nell' $S_{3}(x y z)$ affine (n. 6), scegliamo come punto doppio $B$ il punto improprio dell'asse $x$ e come piano tangente fisso il piano improprio; otteniamo direttamente

$$
\left(\lambda_{0} y+F_{1}\right) x+\lambda_{9} y^{3}+F_{1} y^{2}+F_{2} y+F_{3}=0 \text {. }
$$

Consideriamo infine il caso che le rette $l$ (n. 7) delle $\infty^{2} R_{2}^{(4)}$ di $\Sigma$ coincidano, in una retta fissa $b$. Per due generatrici per un punto generico $B$ della retta $b$ passa una determinata $R_{z}{ }^{(4)}$ di $\Sigma$, che si spezza in un cono eubico $K_{3}{ }^{(2)}$ e in un piano $\beta$ per $b$, fisso al variare di $B$ su $b$. Agli $\infty^{1}$ coni $K_{3}^{(2)}$, i quali formano un fascio, corrisponde sulla $\Phi$ un fascio di cubiche $C^{(3)}$, le quali hanno in comune un punto-base $A_{1}$, sezione del piano $\beta$ colla $\Phi$. Immergiamo l' $S_{r}$ della $V_{3}^{(r+1)}$ in un $S_{r+1}$ e siano $A$ un suo punto generico, ed $\alpha$ il piano che da $A$ proietta la retta $b$. Alle generatrici del cono $K_{3}^{(2)}$, di vertice $B$, facciamo corrispondere proiettivamente i punti della retta $A B$, in modo che alla generatrice $B A_{1}$ corrisponda $A$. Si ottiene così nell' $S_{r+1}$ una rigata $V_{3}$, le cui 
generatrici congiungono il piano $\alpha$ colla $\Phi$, che dal punto $A$ si proietta nella $V_{3}^{(r)}$ data dell' $S_{r}$. E poichè sulla $V_{3}$ dell' $S_{r+1}$ si possono ripetere le considerazioni già fatte in questo $\mathrm{n}^{0}$., essa si puó considerare come proiezione di una $V_{3}^{(13)}$ dell' $S_{12}$, cioè la $V_{3}^{(r+1)}$ stessa è proiezione di tale $V_{3}^{(13)}$.

\section{Capitoto III.}

\section{$F_{3}^{(m)}$ a superficie-sezione rappresentabile nel piano da un sistema lineare di sestiche con 7 punti-base doppi.}

9. Queste $V_{3}^{(n)}$ sono state studiate da Fano $\left({ }^{15}\right)$. Esse sono tutte proiezioni della $V_{3}^{(8)}$ dell' $S_{7}$, sezione completa di un $S_{1}$-cono di VERONEse con una quadrica dell' $S_{7}$. Queste $V_{3}{ }^{(r)}$ sono razionali e rappresentabili nello spazio lineare $S_{3}{ }^{*}$ dal sistema lineare delle $\varphi^{(4)}$, con un tacnodo B, quattro rette-base per $\mathrm{B}$ e una $\mathrm{C}^{(4)}$ base, sezione di una $\varphi^{(4)}$ con un piano generico.

Scegliendo nell' $S_{3}(x, y, z)$ affine (n.6) il punto $B$ nel punto improprio dell'asse $x$, come piano tacnodale il piano improprio e la $C^{(+)}$.base nel piano $(y z)$, si ottiene come rappresentazione analitica di questo sistema lineare di $\varphi^{(4)}$

$$
\lambda_{0} x^{2}+x\left(\lambda_{1} y^{2}+F_{1} y+F_{2}\right)+\lambda_{7}\left(\bar{\lambda}_{0} y^{2}+\bar{F}_{1} y^{3}+\bar{F}_{2} y^{2}+\bar{F}_{3} y+\bar{F}_{4}\right)=0 .
$$

CaPitolo IV.

\section{$V_{3}^{(n)}$ a curve-sezioni iperellittiche di genere $\pi=3$.}

10. Consideriamo nello spazio lineare $S_{\mu},(r \geq 4)$, una $V_{3}^{(v+1)}$ a curvesezioni iperellitiche di genere $\pi=3$. La $V_{3}^{(r+1)}$ contiene un fascio lineare di quadriche $|Q|$, i cui spazi d'appartenenza $S_{3}$ generano una varietà $M_{4}^{(2-3)}$ razionale normale, $\left({ }^{16}\right)$.

Proviamo che per $\mathrm{r} \geq 7$ la $\mathrm{V}_{3}^{(\mathrm{r}+1)}$ è l'intersezione residua della $\mathrm{M}_{4}^{(\mathbf{r}-3)}$ con un' iperquadrica $\mathrm{Q}_{\mathrm{r}-1}$ dell' $\mathrm{S}_{\mathrm{r}}$ che passa per $\mathrm{r}-7$ spazi lineari $\mathrm{S}_{3}$.dell' $\mathrm{M}_{4}^{(\mathbf{r}-3)}$.

Indichiamo con $N$ il numero delle $Q_{n-1}$ linearmente indipendenti per

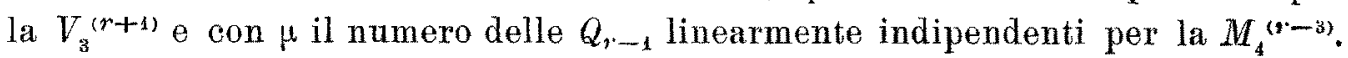
Se la differenza

$$
d=N-\mu>0,
$$

allora esistono $d>0$ iperquadriche $Q_{r_{-1}}$ linearmente indipendenti che passano

(15) FANO G., loc. cit. $\left({ }^{10}\right)$.

(16) ENrIQUes F., Sui sistemi lineari di superficie algebriche ad intersezioni variabili iperellittiche [" Math. Annalen », Bd. 46 (1896); pp. 173-199]. 
per la $V_{3}^{(r+1)}$ senza contenere la $M_{4}^{(r-3)}$. Una di esse $Q^{*}{ }^{*}-i$, sega la $M_{4}^{(r-3)}$ secondo la $V_{3}^{(r+1)}$, ed ulteriormente in una $V_{3}^{(v)}$, dell' ordine $\nu=2(r-3)-$ $-(r+1)=r^{*}-7$. Poichè la $Q^{*}{ }^{*}-1$ sega un $S_{3}$ generico della $M_{4}{ }^{(r-3)}$ nella quadrica $Q$ della $V_{3}^{(r+1)}$ in esso contenuta, la $V_{3}^{(v)}$ si spezza in $v=r-7 \mathrm{di}$ quegli spazi $S_{3}$. La dimensione del sistema lineare completo di queste $V_{3}^{(\nu)}$ riducibili essendo $r-7$, il numero $d$ dato dalla (4) dovrà essere

$$
d \leq r-6 \text {. }
$$

Per calcolare $N$, osserviamo che sopra una superficie-sezione $F_{2}^{(r+1)}$ della $V_{3}^{(r+1)}$ il sistema $|2 F|$ delle sezioni della $V_{3}^{(r+1)}$ colle iperquadriche $Q_{1-1}$ determina un sistema di curve $|2 C|$, doppio del sistema delle curvesezioni $|C|$ della $F$. Il sistema $|2 C|$ ha il grado $n^{\prime}=4(r+1)$ e il genere $\pi^{\prime}=2 \cdot 3+(r+1)-1=r+6$, quindi la sua dimensione è $r^{\prime} \leq n^{\prime}-\pi^{\prime}+$ $+1=3 r-1$. Poichè una $(2 F)$ che contenga $F_{2}^{(r+1)}$ si spezza nella $F_{2}^{(r+1)} \mathrm{e}$ in una generica superficie-sezione della $V_{3}^{(r+1)}$, le $(2 F)$ linearmente indipendenti che contengono la $F_{2}^{(r+1)}$ sono $r+1$; cioè la dimensione $\delta$ del sistema $|2 F|$ è $\delta=r^{\prime}+(r+1) \leq(3 r-1)+(r+1)=4 r$. Il numero delle $Q_{r-1}$ linearmente indipendenti dell' $S_{r}$ essendo $\left(\begin{array}{c}r+2 \\ 2\end{array}\right)$, segue

$$
N=\left(\begin{array}{c}
r+2 \\
2
\end{array}\right)-\delta-1 \leq \frac{1}{2}\left(r^{2}-5 r\right)
$$

Come noto $\left({ }^{17}\right)$

$$
\mu=\left(\begin{array}{c}
r+1 \\
2
\end{array}\right)-2(r-3)=\frac{1}{2}\left(r^{2}-7 r+12\right)
$$

di modo ehe per il numero (4) si ha

$$
d=N-\mu \geq \frac{1}{2}\left(r^{2}-5 r\right)-\frac{1}{2}\left(r^{2}-7 r+12\right)=r-6
$$

e quindi, in base alla (5), $d=r-6$. Dunque la (4) è verificata per $r \geq 7$ (e i vari sistemi lineari considerati sono completi).

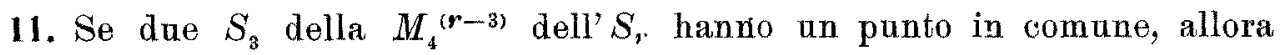
tutti gli $\infty^{t} S_{3}$ della $M_{4}^{(r-8)}$ passano per questo punto, cioè o gli $S_{3}$ della $M_{4}^{(r-3)}$ sono a due a due non incidenti, oppure la $M_{4}^{(r-3)}$ è un cono. Il ver*

(17) MoRIN U., Sui sistemi lineari di superficie algebriche a curva-caratteristica di geneve due ["Annali di Mat. ", t. 19 (4), 1940], pp. 257-288. Aleune considerazioni di questa Memoria sono state direttamente sfruttate in questo capitolo, conservando (per facilitare il confronto) il più possibile anche la forma. 
tice di questo cono può essere un punto, o una retta o un piano $\left(S_{0}-, S_{1}-\right.$ o $S_{2}$-conol.

Per $r>7$ la $M_{4}^{(r-3)}$ non può essere un $S_{2}$-cono. Infatti, l' $S_{n_{-1}}$ d'appar* tenenza di $r-3$ quadriche $Q$ della $V_{3}{ }^{(\boldsymbol{r}+t)}$ taglierebbe questa varietà in una superfioie dell' ordine $2(r-3)>r+1$, ciò che è assurdo.

Se $l^{\prime} M_{4}^{(r-3)}$ è un cono di vertice $A$, poichè per $r \geq 7$ l'iperquadrica $Q_{r-1}$ passa per $r-7$ spazi $S_{3}$ del cono (n. 10 ), per $r>7$ la $Q_{r-1}$ passa per il vertice $A$ del cono, ciò̀ questo punto risulta quadruplo per la $V_{3}^{(r+1)}$. Invece per $r=7$ questo punto sarà quadruplo per la $V_{3}^{\left({ }^{(8)}\right.}$ soltanto nel casu particolare che la $Q_{n_{-1}}$ passi per il vertice del cono $M_{4}^{\left({ }^{(3)}\right.}$.

Dal punto quadruplo $A$ la $V_{3}^{(r+1)}$ si proietta biunivocamente su una $M_{3}^{(r-3)}$ di un $S_{r-1}$ (a curve-sezioni razionali). Risulta così opportuna la sud. divisione dello studio delle $V_{3}^{(r+1)}$ (non coni) di questo capitolo nei seguenti paragrafi :

1) $V_{3}^{(r+1)}$ la cui $M_{4}^{(r-3)}$ non è un cono;

2) $V_{3}^{(r+1)}(r \geq 7)$ con (almeno) un punto quadruplo;

3) $V_{3}^{(8)}$ dell' $S_{\gamma}$ priva di punti quadrupli, la eui $M_{4}^{(4)}$ è un $S_{0}$-cono;

4) $V_{3}^{(7)}$ dell $S_{6}$;

5) $V_{3}^{(6)}$ dell' $S_{5}$;

6) $V_{3}^{(5)}$ dell' $S_{4}$.

\section{§ 1. $V_{3}^{(r+1)}$ la cui $M_{4}^{(r-3)}$ non ̀̀ un cono.}

12. La $\nabla_{3}^{(r+1)}$ si proietta biunivocamente dall' $\overline{S_{3}}$ d' una sua quadrica $Q$ sopra una $V_{3}^{\left(n^{*}\right)}$ d'uno spazio $S_{r^{*}}\left(r^{*}=r-4\right)$, contenente un fascio lineare di quadriche $Q^{*}$, d'ordine $n^{*}=r-5$, ciò̀ a curve-sezioni ellittiche.

Infatti $\left({ }^{18}\right)$, la superficie-sezione della $V_{3}^{(r+1)}$ con un $S_{r-1}$ per l' $\bar{S}_{3}$ di proiezione taglia la $V_{3}^{(r+1)}$ nella quadrica $\bar{Q}$ dell' $\overline{S_{3}}$ ed ulteriormente in una superficie $F_{2}^{(r-1)}$, la quale ha con la $\bar{Q}$ in comune una conica $C^{(2)}$. Un $S_{r-2}$ dell' $S_{,-1}$ per la $C^{(2)}$ taglia la $F_{2}^{(r-1)}$ in questa conica $C^{(2)}$ ed ulteriormente in una curva $C^{(r-3)}$, la quale ha con la $C^{(2)}$ in comune due punti; cioè le curve-sezioni della $V_{3}^{(n *)}$ hanno l'ordine $n^{*}=r-5$. Esse sono normali nel xispettivo $S_{r \rightarrow 7}$ dell' $S_{, *}\left(r^{*}=r-4\right)$ quindi ellittiche.

Una $V_{3}^{(r-5)}$ dell' $S_{r-4}\left(r^{*}=r-4\right)$, a curve-sezioni ellitiche e dotate di un fascio lineare di quadriche, è razionale e può essere rappresentata nell' $S_{3}^{*}$ da

$$
\text { per } r^{*}=4
$$

(18) loe. cit. $(10)$ 
10) sistema di superficie cubiche $\varphi^{(3)}$ determinato da una retta e una quintica, intersezioni totali con una quadrica (rappresentativo di un'ipersu. perficie cubica dell' $S_{4}$ contenente un pianol;

$$
\text { per } r=5,\left({ }^{19}\right) \text {, }
$$

$\left.2^{\circ}\right)$ sistema di $p^{(3)}$ determinato da una quartica di $1^{\text {a }}$ specie ed una sua corda;

$$
\text { per } r^{*}=6,
$$

$\left.3^{0}\right)$ sistema di $\varphi^{(3)}$ determinato da una cubica sghemba ed una retta ad essa semplicemente appoggiata;

$$
\text { per } r^{*}=7
$$

$\left.4^{\circ}\right)$ sistema delle $\varphi^{(3)}$ per tre rette sghembe;

$\left.5^{\circ}\right)$ sistema delle quadriche con due punti-base, eventualmente infinitamente vicini ;

$$
\text { per } 6 \leq r^{*} \leq 9
$$

$\left.6^{\circ}\right)$ sistema di $\varphi^{(3)}$ con un punto-base doppio, $10-r^{*}$ rette-base per esso, ed in esso lo stesso cono quadrico tangente;

$$
\text { per } r^{*}=9 \text {, }
$$

$\left.7^{\circ}\right)$ sistema di $\varphi^{(4)}$ con un punto-base triplo, col cono tangente fisso, $e$ due rette-base doppie per esso.

13. Poiohè nella $V_{3}^{(r+1)}$ il sistema completo delle superficie-sezioni $|F|$ è la somma minima del fascio lineare di quadriche $Q$ e del sistema $|F-Q|$ delle superficie-sezioni della $V_{3}^{(r-5)}$, il sistema lineare di superficie $|\varphi|$ rap. presentativo della $V_{3}^{(r+1)}$ è la somma del sistema rappresentativo della $V_{3}^{(r-5)}$ $e$ del fascio di superficie rappresentativo delle quadriche $Q^{*}$ della $V_{3}^{(r-5)}$. Nei numeri successivi opereremo questa somma per i casi delle diverse

(19) I successivi sistemi sono tratti dalla classificazione dell'Enrouts, Sai sistemi lineari di superficie algebriche ad intersezioni variabili iperellittiche ["Mathem. Annalen ", Bd. $46(1896)]$, dove nel sistema $2^{\circ}$ la $C^{(5)}$-base $\dot{e}$ stata spezzata affinchè la $V_{3}$ contenga un fascio di quadriche. Il sistema $5^{\circ}$ si deduce dai sistemi $5^{0}$ e $6^{\circ}$ dell' Enriquas [sistema di $\varphi^{(3)}$ con una cubica-base gobba e su essa un punto.base $A$ doppio; sistema di $\varphi^{(3)}$ con un puntobase $B$ biplanare $B$, in esso un piano tangente - fisso, passanti per una cubica col punto doppio $B]$, facendo prima degenerare la cubica-base in una retta e una conica $C^{(2)}$ (affinchè la $V^{(3)}$ eon* tenga un fascio di quadriche) e poi trasformando il sistema $\left|\varphi^{(3)}\right|$ mediante la corrispondenza cremoniana definita nell' $S_{3}$ dal sistema omaloidico delle quadriche per la conica $C^{\gamma(2)}$ e rispet. tivamente per il punto $A$ oppure col piano tangente $\tau$ fisso in $B$. 
$V_{3}^{(r-5)}$ elencate al numero precedente, e descriveremo direttamente la $V_{3}^{(r+1)}$ dell' $S_{r}$ così rappresentata.

14. $1^{\circ} \mathrm{C}_{\mathrm{ASO}}: \mathrm{V}_{3}^{(9)}$ dell' $\mathrm{S}_{8}$ rappresentata nell' $\mathrm{S}_{3}{ }^{*}$ dal sistema lineare di $\varphi^{(1)}$ con una retta-base b doppia e una $\mathrm{C}^{(5)}$-base, sezione residua di una $\varphi^{(3)}$ e una $\varphi^{(2)}$ con la retta semplice b.

Per dare di questo sistema lineare di $\varphi^{(4)}$ la rappresentazione analitica nell' $S_{3}(x, y, z)$ affine (n. 6) faremo (qui e in tutto questo capitolo) la convenzione che la retta $b$ sia la retta impropria del piano $(x y)$. Si può allora scrivere direttamente l'equazione del sistema di $\varphi^{(t)}$

$$
\begin{gathered}
\left(\bar{F}_{1} x+\bar{G}_{1} y+\bar{F}_{2}\right)\left(F_{1} x+G_{1} y+F_{2}\right)+ \\
+\left(\bar{H}_{1} x^{2}+\bar{L}_{1} x y+\bar{M}_{1} y^{2}+\bar{H}_{2} x+\bar{L}_{2} y+\bar{F}_{3}\right) H_{1}=0
\end{gathered}
$$

15. $2^{\circ}$ CASO: $V_{3}^{(10)}$ dell' $S_{y}$ rappresentata nell' $S_{3}^{*}$ dal sistema lineare di $\varphi^{(4)}$ con una retta-base doppia b e una $\mathrm{C}^{(1)}$-base semplice. di $1^{\mathrm{a}}$ specie, di cui b $\grave{e}$ una corda; rappresentato nell' $S_{3}(x, y, z)$ dall' equazione

$$
\begin{gathered}
\left(\bar{F}_{1} x+\bar{G}_{1} y+\bar{F}_{2}\right)\left(F_{1} x+G_{1} y+F_{2}\right)+ \\
+\left(\bar{\lambda}_{0} x^{2}+\bar{\lambda}_{1} x y+\bar{\lambda}_{2} y^{2}+\bar{H}_{1} x+\bar{L}_{1} y+\bar{G}_{2}\right) G_{2}=0 .
\end{gathered}
$$

16. $3^{\circ}$ CAso: $\mathrm{V}_{3}^{(11)}$ dell $\mathrm{S}_{10}$ rappresentata nell' $\mathrm{S}_{3}{ }^{*}$ dal sistema delle $\varphi^{(4)}$ con una relta-base b doppia e una cubica sghemba ad essa semplicemente appoggiata. Se nell' $S_{3}(x, y, z)$ diamo della cubica le seguenti equazioni parametriche $x=\frac{1}{t^{3}-t}, y=\frac{1}{t^{2}-1}, z=\frac{t}{t^{2}-1}$, il sistema di $\varphi^{(3)}$ è dato dalla seguente equazione

$$
\begin{gathered}
(x y-z) F_{1}+\lambda_{2}\left(x^{2} z-y^{2}\right)+ \\
+(y z-y+1)\left(\lambda_{3} x+\lambda_{4} y+\lambda_{5} z+\lambda_{6}\right)=0 .
\end{gathered}
$$

17. $4^{\circ}$ CAso: $V_{3}{ }^{(12)}$ dell' $\mathrm{S}_{11}$ rappresentata nell' $\mathrm{S}_{3}$ dal sistema delle $\varphi^{(t)}$ con una retta-base doppia b e due rette-base semplici sghembe. L'equazione di questo sistema lineare di ' $\rho^{(4)}$ nell' $S_{3}(x, y, z)$ eे [scegliendo (n. 14) come retta $b$ la retta impropria del piano (xy) e come rette-base semplici l'asse $z$ e la retta di equazioni $y-1=0, x+z-1=0$ ]

$$
\begin{gathered}
(y-1)\left(\lambda_{0} x+\lambda_{1} y\right)+(x+z-1)\left(\lambda_{2} x+\lambda_{3} y\right)+ \\
+z\left[(y-1)\left(\lambda_{4} x+\lambda_{5} y\right)+(x+z-1)\left(\lambda_{6} x+\lambda_{7} y\right)\right]+ \\
+z^{2}\left[(y-1)\left(\lambda_{8} x+\lambda_{9} y\right)+(x+z-1)\left(\lambda_{10} x+\lambda_{11} y\right)\right]=0 .
\end{gathered}
$$


18. $5^{\circ}$ CASO: $\nabla_{3}{ }^{(12)}$ dell' $S_{11}$ rappresentala nell' $\mathrm{S}_{3}$ dal sistema delle $\varphi^{(3)}$ con una relta-base b e su questa due punti-base doppi (eventualmente infinita* mente vicini). L' equazione di questo sistema di $\varphi^{(3)}$ nell' $S_{3}(x, y, z)$ è (n. 14)

$$
F_{1}\left(\bar{\lambda}_{3} x^{2}+\bar{\lambda}_{1} x y\right)+F_{2} x+G_{2} y+F_{3}=0 \text {. }
$$

19. $6^{\circ}$ CASO: $\nabla_{3}^{(14-\nu)}$ dell' $\mathrm{S}_{13-\nu}(0 \leq \nu \leq 3)$ rappresentata nell' $\mathrm{S}_{3}{ }^{*}$ dal sistema di $\varphi^{(i)}$ con un punto-base triplo, in cui il cono tangente si spezza in un cono quadrico fisso e un piano variabile per una generatrice b del cono, la quale è doppia per le $\varphi^{(+)}$; ed inoltre $\vee$ goneratrici come rette-base semplici. Per $y=0$, l'equazione di questo sistema di $\varphi^{(j)}$ nell' $S_{3}(x, y, z)$ affine (n. 14) è (scegliendo inoltre come punto-base triplo il punto improprio dell'asse $x$ )

$$
\left(\bar{F}_{1} y+\bar{F}_{2}\right) F_{1} x+F_{2} y^{2}+F_{3} y+F_{4}=0 \text {. }
$$

20. $7^{\circ}$ CAso: $\mathrm{V}_{3}^{(14)}$ dell' $\mathrm{S}_{1: 3}$ rappresentata nell' $\mathrm{S}_{3}{ }^{*}$ dal sistema di $\varphi^{(5)}$ con un punto-base quadruplo, una relta-base tripla b e una doppia c per esso, ed inoltre il cono tangente spezzato nel piano (bc), in un cono quadrico fisso $e$ in un piano variabile per b. Li equazione di questo sistema di $\varphi^{(5)}$ nel. l' $S_{3}(x, y, z)$ affine (n. 14), scelta inoltre come retta-base $c$ la retta impropria del piano $(x z)$, ̀̀

$$
F_{1}\left(\bar{F}_{1} y+\bar{G}_{1}\right) x+F_{3} y^{2}+G_{3} y+H_{3}=0
$$

\section{§ 2. $V_{3}^{(r+1)}$ dell's. $(r \geq 7)$ con un punto quadruplo $A$.}

21. Nell'ipotesi di questo paragrafo la $M_{4}^{(r-3)}$, luogo degli $\infty^{1}$ spazi $S_{3}$ delle quadriche $Q$ della $V_{3}^{(r+1)}$, è un cono (n. 11), il eui vertice è il punto $A$ (oppure una retta $\alpha$ passante per $A$, od anche, per $r=7$ (n. 11), un piano $\alpha$ passante per $A$ ). La $V_{3}^{(r+1)}$ è l'intersezione residua del cono $M_{4}^{(r-3)}$ con un' iperquadrica $Q_{r-1}$ passante per $h=r-7$ spazi $S_{3}^{\left({ }^{(1)}\right.}, \ldots, S_{3}^{(h)}$ dell' $M_{4}^{(r-3)}$, (n. 10). Per $r>7$ la $Q_{r-1}$ passa quindi per il vertice $A$ del cono $M_{4}^{\left({ }^{(0-3)} \text {, il }\right.}$ quale è quindi quadruplo per la $V_{3}^{(r+1)}$; invece per $r=7$ il punto $A$ sarà quadruplo per la $V_{3}^{(r+1)}$ se facciamo la particolare ipotesi che la $Q_{r-1}$ passi per $A$ (n. 11).

Ún iperpiano generico $S^{*}{ }_{r-1}$ dell' $S_{r}$ sega il cono $M_{4}^{(r-3)}$ in una varietà $M_{3}^{(r-3)}$ luogo di $\infty^{1}$ piani, ed in particolare gli spazi $S_{3}^{(1)}, \ldots, S_{3}^{(h)}(h \geq 0)$ nei piani $\alpha^{(1)}, \ldots$, $\alpha^{(h)}$. La $Q_{r-1}$ viene segata dall' $S_{r-1}^{*}$ in una $Q^{*}{ }_{r-2}$, passante per i piani $\alpha^{(1)}, \ldots, \alpha^{(h)}$.

Dal punto quadruplo $A$ la $V_{3}^{(r+1)}$ si proietta biunivocamente sulla $M_{3}^{(r-3)}$ dell' $S^{*}{ }_{r-1}$. La $V_{3}^{(r+1)}$ ò rappresentata nella $M_{3}^{(r-3)}$ dal sistema lineare di 
superficie $|\Phi|$, determinato dalle sezioni variabili dell' $M_{3}^{(r-3)}$ colle iperqua. driche dell' $S_{r-1}^{*}$ che passano per i piani $\alpha^{(1)}, \ldots, \alpha^{(h)}$ e per la curva $C^{(\mu)}$, traccia sull' $S_{r-1}^{*}$ del cono $K_{z}^{(r+1)}$ secondo il quale $l^{\prime} S_{r-1}$ tangente alla $Q_{r-1}$ nel punto $A$ sega la $V_{3}^{(r+1)}$ (il quale $S_{r \rightarrow 1}$ contiene gli spazi $S_{3}^{(1)}, \ldots, S_{3}^{(h)}$ ).

Infatti un punto-base del sistema $|\Phi| \grave{e}$ proiettato da $A$ in una retta appartenente alla $V_{3}^{\left({ }^{2}+1\right)}$, cioè al cono $K_{2}^{\left({ }^{r+1)}\right.}$ (e viceversa). Inoltre la $F^{(r+1)}$ sezione della $V_{3}^{(r+1)}$ coll' $S^{*}{ }_{r-1}$ è segata sulla $M_{3}^{\left(r^{\prime}-1\right)}$ dall' iperquadrica $Q^{*}{ }_{r-2}$ dell' $S^{*}{ }_{r-1}$ passante per i piani $\alpha^{(1)}, \ldots, \alpha^{(h)}$. Dunque la $C^{(\mu)}$ è la sezione della $F^{\left(r^{+}+1\right)}$ con un $S_{r-2}^{*}$ dell' $S^{*}{ }_{r-1}$ passante per i piani $\alpha^{(1)}, \ldots, \alpha^{(h)}$.

La $M_{3}^{(r-3)}$ può venire rappresentata in uno spazio lịeare $S_{3}^{*}$ da un sistema lineare di superficie $\varphi^{(s)}$ (di un certo ordine $s$ ), con una retta-base $b$ dell'ordine $s-1$, ed altri elementi base. Un sistema lineare di superficie rappresentativo della $V_{3}^{(\mathrm{r}+1)}$ è allora dato dalle $\left|\varphi^{(\mathbf{n})}\right| \equiv\left|2 \varphi^{(\mathbf{s})}-\beta_{1}-\ldots-\beta_{\mathrm{h}}\right|$, (indicando con $\beta_{1}, \ldots, \beta_{\mathrm{h}} \mathrm{h}$ piani per b), con ulteriormente una $\mathrm{C}^{(v)}$-base, sezione di una $\varphi^{(n)}$ con una superficie del sistema $\left|\varphi^{(\mathrm{s})}-\beta_{\mathrm{A}}-\ldots-\beta_{\mathrm{h}}\right|$.

Nei successivi numeri deduremo con questo procedimento il sistema $\left|\varphi^{(n)}\right|$ dal sistema $\left|\varphi^{(s)}\right|$ rappresentativo di una $M_{3}^{(r-3)}$, a partire da $r=7$. Vedremo che soltanto per $r \leq 11$ il $\left|\varphi^{(n)}\right|$ è rappresentativo di una $V_{3}^{(r+1)}$, a curvesezioni iperellittiche di genere $\pi=3$ non cono (e con qualche restrizione per $r \geq 10$ ).

22. Per $r=7$, la $M_{3}{ }^{(4)}$ dell' $S_{6}^{*}$ può essere rappresentata nell' $S_{3}{ }^{*}$ da $\left({ }^{20}\right)$ : I) sistema di $\varphi^{(2)}$ per una retta $b$;

II) sistema di $\varphi^{(3)}$ con due rette-base $b$ e $c$, rispettivamente doppia $\theta$ semplice, incidenti in un punto $B$, in eui sono fissi $i$ due piani tangenti;

III) sistema di $\varphi^{(3)}$ con due rette-base infinitamente vicine $b$ e $c$, rispettivamente doppia e semplice, incidenti in un punto $B$, in cui sono fissi $i$ due piani tangenti;

IV) sistema di $\varphi^{(4)}$ con una retta-base tripla $b$ e lungo essa i tre piani tangenti fissi (ciò̀ tre rette-base $c, d$, e semplici, complanari con $b$ e ad essa infinitamente vicine).

(20) SEqRe C, Sulle varieta normali a tre dimensioni composte di serie semplici razio. nali di piani ["Atti Ace. di Torino", t. 21 (1885-86); pp. 95.115]. Questi sistemi lineari si possono ottenere con procedimento diretio nel seguente modo: I) Se la $M_{3}{ }^{(4)}$ non è un $S_{\text {, }}$-cono, essa si proietta da un suo piano biunivocamente sopra un $S_{3}$, ai piani dell' $M_{3}{ }^{(4)}$ corrispondono i piani per una retta $b$, cioè la $M_{3}^{(4)}$ è rappresentata dalle $\varphi^{(2)}$ per $b$. Se la $M_{3}^{(\xi)}$ è un $S_{0}$-cono, tenuto conto che una rigata $F_{2}^{(4)}$ è rappresentata nel piano dalle II) cubiche con un punto doppio e. uno semplice, III) eubiche con una tangente fissa in un punto doppio, IV) quartiche con tre tangenti fisse in un punto triplo; si ottengono gli altri sistemi lineari di superficie rappresentativi di coni $M_{3}{ }^{(4)}$. 
In conseguenza (n. 21) si hanno i seguenti quattro tipi di $V_{3}^{(8)}$ dell' $S_{7}$, dei quali il III) si può considerare come un caso particolare del II):

I) In questo caso la $M_{4}^{(4)}$ dell' $S_{7}$ è un $S_{0}$-cono, nel quale la $V_{3}^{(8)}$ è segata da una quadrica $Q_{b}$ dell' $S_{\gamma}$ che passa (per ipotesi) per il vertice $A$ del cono. La $V_{3}^{(8)}$ risulta (n. 21) rappresentata nell' $S_{3}{ }^{*}$ dal sistema delle $\varphi^{(*)}$ con una retta-base $\mathrm{b}$ doppia e una $\mathrm{C}^{(6)}$-base, sezione d'mna $\varphi^{(t)}$ con una $\varphi^{(2)}$ per la retta b.

La rappresentazione analitica di questo sistema di $\varphi^{(4)}$ nell' $S_{3}(x, y, z)$ affine (n. 14) è

$$
\begin{gathered}
\left(\bar{F}_{1} x+\bar{G}_{1} y+\bar{F}_{2}\right)\left(F_{1} x+G_{2} y+F_{2}\right)+ \\
+\lambda_{7}\left(\bar{G}_{2} x^{2}+\bar{H}_{2} x y+\bar{L}_{2} y^{2}+\bar{M}_{3} x+\bar{N}_{3} y+\bar{F}_{4}\right)=0 ;
\end{gathered}
$$

III) In questo caso la $M_{4}^{(t)}$ dell' $S_{7}$ è un $S_{4}$-cono (il cui vertice sia una retta a). Allora il punto quadruplo $A$ della $V_{3}^{(8)}$ segata sull' $M_{4}^{(4)}$ da una $Q_{6}$, è uno dei due punti d'incontro della $Q_{6}$ con $a$; oppure, nel caso che la $Q_{6}$ passa per $a$, è un punto generico di questa retta. La $V_{3}^{(8)}$ risulta (n. 21) rappresentata nell' $S_{3}^{*}$ da un sistema lineare di $\varphi^{(6)}$ con le rette $b$ e $c$, rispettivamente quadrupla e doppia, incidenti in un punto $B$, in cui vi sono due piani tangenti tacnodali $\beta, \gamma$, ed inoltre una $C^{(8)}$-base, sozione di una $\varphi^{(\varepsilon)}$ con una $\varphi^{(3)}$ con le rette $b$ e $c$ rispettivamente doppia e semplice, e in $B$ i piani tangenti $\beta$ e $\gamma$.

Mediante la trasformazione cremoniana definita nell' $S_{3}^{*}$ dal sistema omaloidico delle quadriche colle rette-base $b$ e $c$ ed un punto-base sulla $C^{(8)}$, questo sistema di $\varphi^{(6)}$ si trasforma in un sistema lineare di $\varphi^{(5)}$ con due rettebase $\mathrm{b}$ e c, rispettivamente tripla e semplice, incidenti in un punto $\mathrm{B}$, con ivi un piano tangente tacnodale $\beta$ (diverso dal piano bo), ed inoltre con una $\mathrm{C}^{(7)}$ base semplice, seaione di una $\varphi^{(5)}$ con una quadrica per $b$, col piano tangente $\beta$.

Per dare di questo sistema di $\varphi^{(5)}$ la rappresentazione analitica nell' $S_{3}(x, y, z)$ affine (n. 14), supponiamo che la retta $c$ sia rappresentata nel piano improprio dall' equazione $\bar{\lambda}_{1} y+\bar{\lambda}_{2} z=0$. Si ottiene allora

$$
\begin{gathered}
\left(\bar{\lambda}_{0} \bar{F}_{1} x+\bar{G}_{1} y+\bar{F}_{2}\right)\left[\lambda_{0} \bar{F}_{1} x+G_{1} y+F_{2}+\lambda_{6}\left(\bar{\lambda}_{1} y+\bar{\lambda}_{2} z\right) z^{2}\right]+ \\
+\lambda_{7}\left[\bar{\lambda}_{0} \bar{F}_{1}^{2} x^{2}+\bar{F}_{1} x\left(\bar{G}_{1} y+\bar{H}_{2}\right)+\bar{G}_{2} y^{2}+\bar{G}_{3} y+\bar{H}_{4}+\right. \\
\left.+\left(\bar{\lambda}_{1} y+\bar{\lambda}_{2} z\right)\left(\bar{\lambda}_{3} \bar{F}_{1} x+\bar{\lambda}_{4} y z+\bar{\lambda}_{5} z^{2}\right) z^{2}\right]=0 .
\end{gathered}
$$

Se in particolare in questa equazione si pone $\bar{\lambda}_{0}=0$, il sistema lineare di $\varphi^{(5)}$ rappresenta una $V_{3}^{(8)}$ dell' $S_{\gamma}$ per la quale la retta $a$ (vertice del cono $M_{4}^{(4)}$ ), è quadrupla (cioè la quadrica $Q_{6}$, che segna nell' $M_{4}^{(4)}$ la $V_{3}^{(8)}$, passa per questa retta). 
III) II sistema lineare rappresentativo della $V_{3}^{(8)}$ relativa a questo caso è un caso particolare del sistema (14), con $\ddot{\lambda}_{1}=0$.

IV) In questo caso il vertice del cono $M_{4}^{(t)}$ è un piano $\alpha$ e la conica, sezione di questo piano colla quadrica $Q_{6}$, è quadrupla per la $V_{3}^{(8)}$. La $V_{3}^{(8)}$ risulta (n. 21) rappresentata nell' $S_{3}^{*}$ dal sistema lineare delle $\varphi^{(8)}$ con una retta sestupla $b$, tre rette doppie $c, d$, e rispettivamente complanari con $b$ e ad essa infinitameate vicine, ed una $C^{(8)}$-base, sezione di una $\varphi^{(8)}$ con una $\varphi^{(1)}$ con la retta tripla $b$ e le rette semplici $c, d, e$.

Mediante la corrispondenza cremoniana definita nell' $S_{3}^{*}$ dal sistema omo* loidico delle $\varphi^{(t)}$ con la retta tripla $b$, le rette sempliçi $c, d$, e e tre puntibase sulla $C^{(8)}$, il sistema di $\varphi^{(8)}$ si trasforma nel sistema lineare di $\varphi^{(5)}$ con una rella-base b tripla, tre piani tangenti fissi lungo la $\mathrm{b}$, ed una $\mathrm{C}^{(5)}-b a s e$, sezione di una $\varphi^{(5)}$ con un piano generico dell' $\mathrm{S}_{3}^{*}$.

Per dare di questo sistema di $\varphi^{(5)}$ la rappresentazione analitica nell' $S_{3}(x, y, z)$ affine (n. 14), scegliamo la $C^{(5)}$-base nel piano $(x, z)$. Si ottiene allora

$$
\bar{F}_{3}\left(\lambda_{0} x^{2}+\lambda_{1} x y+\lambda_{2} y^{2}\right)+\lambda_{0}\left(\bar{F}_{4} x+\bar{F}_{\mathrm{5}}\right)+F_{4} y=0
$$

73. Una $V_{3}^{\left({ }^{(9)}\right.}$ dell' $S_{8}^{S_{3}}$ con un punto quadruplo $A$ è (ove esista) la sezione residua di un cono $M_{4}{ }^{(5)}$ dell' $S_{8}$ con una quadrica $Q_{7}$ che passa per uno spazio $S_{3}^{\left({ }^{(1)}\right.}$ del cono $M_{4}^{\left({ }^{5}\right)}$ (n. 10). Viceversa, una $Q_{7}$ generica dell' $S_{8}$ che passi per uno spazio $S_{3}{ }^{\left({ }^{1}\right)}$ del cono $M_{4}^{(5)}$ (il cui vertice non sia un piano, n. 11), sega gli $\infty^{1} S_{3}$ dell' $M_{4}{ }^{(5)}$. nelle $\infty^{1}$ quadriche $Q$ di una $V_{3}^{\left({ }^{(9)}\right.}$.

La $M_{3}{ }^{(5)}$, sezione dell' $M_{4}^{\left({ }^{(5)}\right.}$ con un $S_{7}^{*}$ generico dell' $S_{8}$ (n. 21), può essere rappresentata nell' $S_{3}^{*}$ da un sistema lineare di $\left({ }^{21}\right)$ :

I) $\varphi^{(3)}$ colle rette-base $b$ e $c$ incidenti, rispettivamente doppia e semplice;

II) $\varphi^{(3)}$ colla retta doppia $b$ e lango essa il piano tangente $\gamma$ fisso;

III) $\varphi^{(3)}$ con una retta doppia $b$ in un punto $B$ di essa fissi $i$ due piani tangenti;

IV) $\varphi^{(t)}$ con una retta tripla $b$, due piani tangenti $\gamma$ e $\delta$ fissi per essa, ed in un suo punto $B$ fisso anche il terzo piano tangente.

(i1) Se la $M_{3}^{(5)}$ non è un cono, essa si proietta da un suo piano sopra una $M_{3}^{(2)}$ dell' $S_{4}$ (lungo di $\varsigma^{ \pm}$piani), rappresentata nell' $S_{3}$ dalle quadriche per due rette incidenti $b$ e oppure dalle quadriche con un piano tangente $\gamma$ fisso lungo nna retta $b$. Sommando a questi

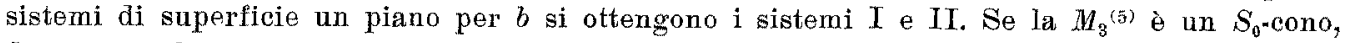
dai sistemi lineari di curve rappresentativi in un piano di una $F_{2}{ }^{(5)}$ dell' $S_{6}$, cioè IrI) cubiche con un punto doppio, IV) quartiche con due tangenti fisse in un punto triple; si ricavano gli ultimi due sistemi di superficie rappresentativi di un $S_{0}$-cono $M_{3}{ }^{(5)}$, 
In conseguenza (n. 21) si hanno i seguenti quattro tipi di $V_{3}{ }^{(9)}$ dell' $S_{8}$, dei quali il II) si puo interpretare come un caso particolare del I):

I) $V_{3}{ }^{(9)}$ dell' $S_{8}$ rappresentata nell' $S_{3}^{*}$ dal sistema lineare delle $\varphi^{(5)}$ colle rette-base incidenti, $b$ e $\dot{c}$, rispettivamente tripla e doppia, e una $C^{(5)}$-base sezione di una $\varphi^{(5)}$ con una $\varphi^{(2)}$ passante per $b$ e $c$. Mediante la corrispondenza cremoniana definita dal sistema omoloidico delle $\varphi^{(2)}$ per $b, c$ ed un puntobase sulla $C^{(5)}$, il sistema di $\varphi^{(3)}$ si trasforma in un sistema $d i \varphi^{(1)}$ con le rette-base incidenti $\mathrm{b}$ e $\mathrm{c}$, rispettivamente doppia e semplice, ed una $\mathrm{O}^{(4)}$-base, sezione di una $\varphi^{(t)}$ con un piano generico dell' $S_{3}{ }^{*}$.

Per dare di questo sistema di $\varphi^{(1)}$ la rappresentazione analitica nelI' $S_{3}(x, y, z)$ affine (n. 14), scegliamo la $C^{(4)}$-base nel piano (yz) e la rettabase $c$ sia rappresentata nel piano improprio dall' equazione $\bar{\lambda}_{\vartheta} y+\bar{\lambda}_{1} z=0$. Si ottiene allora

$$
\begin{gathered}
\lambda_{0} z^{2}\left(\bar{\lambda}_{0} y+\bar{\lambda}_{1} z\right) x+F_{1} x^{2}+G_{1} x y+F_{2} x+ \\
+\lambda_{8}\left[z^{2}\left(\bar{\lambda}_{0} y+\bar{\lambda}_{1} z\right)\left(\lambda_{2} y+\bar{\lambda}_{3} z\right)+\bar{H}_{1} y^{2}+\bar{G}_{2} y+\bar{H}_{3}\right]=0 .
\end{gathered}
$$

II) Il sistema lineare rappresentativo della $V_{3}{ }^{\left({ }^{9}\right)}$ relativa a questo caso è il caso particolare del sistema $(16), \operatorname{con} \bar{\lambda}_{0}=0$.

III) $V_{3}{ }^{\left({ }^{9}\right)}$ dell' $S_{8}$ rappresentata nell' $S_{3}^{*}$ dalle $\varphi^{(5)}$ con una retta tripla b, un punto $\mathrm{B}$ di questa retta quadruplo ed in esso due piani tangenti fissi per la retta $\mathrm{b}$; ed una $\mathrm{C}^{(7)}$-base, sezione di una $\varphi^{(5)}$ con un cono quadrico di vertice $\mathrm{B}$ e con la generatrice $\mathrm{b}$.

Diamo di questo sistema lineare di $p^{(5)}$ la rappresentazione analitica nell' $S_{3}(x, y, z)$ affine (n. 14), avendo scelto il punto $B$ nel punto improprio dell'asse $x$.

(17) $\left[\bar{F}_{1} y+\bar{F}_{2}\right]\left[\lambda_{0} \bar{G}_{2} x+F_{2} y+F_{3}\right]+\lambda_{8}\left[\bar{G}_{2} x\left(\bar{Q}_{1} y+\bar{H}_{2}\right)+\bar{F}_{3} y^{2}+\bar{F}_{4}^{\prime} y+\bar{F}_{5}\right]=0$.

IV) $V_{3}{ }^{(9)}$ dell' $S_{8}$ rappresentata nell' $S_{3}{ }^{*}$ dal sistema lineare delle $\varphi^{(7)}$ con una retta quintupla $b$, due rette-base doppie $c, d$, infinitamente vicine alla $b$ $\Theta$ ad essa incidenti in un punto sestuplo $B$, con tre piani tangenti fissi per la retta $b$ (di cui due per $c$ e $d$ ); ed inoltre una $C^{(2)}$-base, sezione di una $\varphi^{(7)}$ con una $\varphi^{(3)}$, colla retta doppia $b$, le rette semplici $c, d$ e il punto triplo $B$.

Mediante la corrispondenza cremoniana definita nell' $S_{3}^{*}$ dal sistema oma loidico delle $\varphi^{(3)}$, con la retta doppia $b$, le rette semplici $c, d$ e due punti-base sulla $C^{(7)}$, questo sistema di $\varphi^{(7)}$ si trasforma nel sistema di $\varphi^{(5)}$ con una retta tripla b, lungo essa fissi due piani tangenti, e in un suo punto quadruplo $\mathrm{B}$ fisso anche un terzo piano tangente per $\mathrm{b}$; ed inoltre una $\mathrm{C}^{(5)}$-base, sezione di una ${ }^{(5)}$ con un piano generico per $\mathrm{B}$. 
Come rappresentazione analitica di questo sistema di $\varphi^{(5)}$ nell' $S_{3}(x, y, z)$ affine (n. 14), scelto il punto quadruplo $B$ nel punto improprio dell'asse $x$, $e$ la $C^{(5)}$ nel piano $(x z)$, si ottiene

$$
\bar{F}_{2}\left(\lambda_{0} \bar{F}_{1} x y+F_{1} y^{2}\right)+F_{4} y+\lambda_{8}\left(\bar{F}_{2} \bar{F}_{1} G_{1} x+\bar{F}_{5}\right)=0 \text {. }
$$

24. Una $V_{3}^{(10)}$ dell' $S_{9}$ (non cono) con un punto quadruplo $A$ è (se esiste) l'intersezione residua di un cono $M_{4}^{\left({ }^{6}\right)}$ dell' $S_{9}$ (il cui vertice è il punto $A$ oppure una retta passante peri $A$ ) con una quadrica $Q_{8}$ (non cono di vertice $A$ ) che passa per due spazi, $S_{3}^{(1)}$ e $S_{3}{ }^{(2)}$, del cono $M_{4}{ }^{6}$ (n. 10). Viceversa, una qua. drica $Q_{8}$ che passi per due spazi, $\dot{S}_{3}^{(1)}$ e $S_{3}^{(2)}$, del cono $M_{4}^{(0)}$, non passa di conseguenza per alcun altro punto dello spazio e segna negli $\infty^{\prime} S_{3}$ dell' $M_{4}^{\left({ }^{(b)}\right.}$ le $Q$ di una $V_{3}^{(10)}$.

La $M_{3}^{(6)}$, sezione dell' $M_{4}{ }^{(6)}$ con un $S_{8}^{*}$ generico dell' $S_{\mathrm{g}}$, può essere rappresentata nell' $S_{3}^{*}$ da un sistema lineare di $\left({ }^{22}\right)$.

I) $\varphi^{(3)}$ con una retta-base doppia $b$ e un punto-base semplice $B$;

II) $\varphi^{(3)}$ con una retta-base doppia $b$ e in un suo panto $B$ fisso un piano tangente;

III) $\varphi^{\left.()^{3}\right)}$ con una retta tripla $b$ e lungo essa due piani tangenti fissi;

IV) $\varphi^{(4)}$ con una retta tripla $b$ e una retta-base semplice $c$, incidente la $b$ in an punto $B$, in cai sono fissi i tre piani tangenti;

V) $\varphi^{(4)}$ con una retta tripla $b$, lungo la quale un piano tangente è fisso e in suo punto $B$ sono fissi tutti e tre $i$ piani tangenti;

VI) $\varphi^{(5)}$ con una retta quadrupla $b$, lungo la quale sono fissi tre piani tangenti, $\theta$ in un suo punto $B$ fisso anche il quarto piano tangente.

In conseguenza (n. 21) si hanno i seguenti sei tipi di $V_{3}^{(10)}$ dell' $S_{8}$; dei quali il II si può interpretare come un caso particolare del $I$, e il V come un caso particolare del IV:

I) $V_{3}^{(10)}$ dell' $S_{3}$ rappresentata nell' $S^{*}$ dal sistema lineare delle $\varphi^{(4)}$ con una retla doppia $\mathrm{b}$, un punto-base doppio $\mathrm{B}$, e una $\mathrm{C}^{(4)}$-base, sezione di una $\varphi^{(4}$ con un piano generico per $\mathrm{B}$.

(22) Se la $M_{3}^{(6)}$ non è un $S_{0}$ cono, essa si proietta da un suo piano in ana $M_{3}^{(3)}$ nell' $S_{5}$, rappresentata nell' $S_{3}$ da: I) $\varphi^{(2)}$ con una rettabase $b$ e un punto-base $B$; II) $\varphi^{(2)}$ con una retta-base $b$ in un suo punto $B$ il piano tangente fisso; III) $\varphi^{(3)}$ con una retta doppia $b$ e lungo essa i due piani tangenti fissi. Sommando a questi sistemi un piano per $b$, si otten. gono i primi tre sistemi rappresentativi di $M_{3}^{(6)}$. Se la $M_{8}^{(6)}$ è un $S_{8}$-cono, tenuto conto che una $F_{2}^{(6)}$ non cono dell' $S_{7}$ è rappresentata nel piano da un sistema lineare di: IV) quartiche con un punto-base triplo e uno semplice: $V$ ) quartiche con in un punto-base triplo una tan. gente fissa; VI) quintiche con in un punto-base quadruplo tre tangenti fisse; si ottengono gli ultimi tre sistemi lineari di superficie rappresentativi di $M_{3}^{(\hat{b})}$. 
Per dare di questo sistema di $\varphi^{(4)}$ la rappresentazione analitica neIl' $S_{3}(x, y, z)$ affine (n. 14), soegliamo il punto doppio $B$ nell'origine e la $C^{(4)}$. base nel piano $(x y)$. Si ottiene allora

$$
F_{2} x^{2}+G_{2} x y+H_{2} x z+\lambda_{9}\left[L_{2} y^{2}+\bar{M}_{2} y z+\bar{N}_{2} z^{2}\right]=0 .
$$

II) $V_{3}^{(10)}$ dell' $S_{9}$ rappresentata nell' $S_{3}^{*}$ dal sistema lineare delle ${ }^{(4)}$ con una retta doppia $\mathrm{b}$, su essa un tacnodo $\mathrm{B}$, e una $\mathrm{C}^{(4)}$-base sezione $d^{\prime}$ una $\varphi^{(4)}$ con un piano generico per B.

Scelto il punto $B$ nel punto improprio dell' asse $x$ e la $C^{(4)}$ nel piano $(x y)$, si ottiene come rappresentazione analitica nell' $S_{3}(x, y, z)$ affine (n. 4$)$ di questo sistema di $\varphi^{(t)}$,

$$
\bar{F}_{1} F_{1} x y+F_{2} y^{2}+F_{3} y+\lambda_{9}\left[\bar{F}_{1}^{2} x^{2}+\bar{F}_{1} \bar{F}_{2} x+\bar{F}_{4}\right]=0
$$

III) $V_{3}^{(10)}$ dell' $S_{9}$ rappresentata nell' $S_{3}^{*}$ dal sistema lineare delle $\varphi^{(6)}$ con una retta quadrupla $b$ e due rette doppie $c, d$, incidenti la $b$ è ad essa infinitamente vicine; e due coniche-base in due piani per la retta $b$.

Mediante la trasformazione cremoniana definita nell' $S_{3}^{*}$ dal sistema delle $\varphi^{(3)}$ con la retta doppia $b$, le rette semplici $c, d$ ed un punto-base su cia. scuna delle due coniche-base del sistema di $\varphi^{(6)}$, questi si trasforma nel sistema di $\varphi^{(1)}$ con una retta doppia b ed aventi tra di loro un contatto del secondo ordine lungo due piani tangenti fissi per questa retta.

Scelti nell' $S_{3}(x, y, z)$ affine (n. 14) come piani tangenti fissi alle $\varphi^{(4)}$ il piano $(x y)$ e il piano improprio, si ottiene la seguente rappresentazione analitica

(21) $z\left(F_{1} x+G_{1} y\right)+F_{4}+\lambda_{9}\left[\bar{\lambda}_{0} x^{2}+\bar{\lambda}_{1} x y+\bar{\lambda}_{2} y^{3}+x\left(\bar{\lambda}_{3} z^{3}+\bar{\lambda}_{4}\right)+y\left(\bar{\lambda}_{5} z^{3}+\bar{\lambda}_{6}\right)\right]=0$.

IV) $V_{3}^{(10)}$ dell' $S_{3}$ rappresentata nell' $S_{3}^{*}$ dal sistema lineare delle $\varphi^{(6)}$ con le rette-base $b$ e $c$, rispettivamente quadrupla $\theta$ doppia, incidenti in un punto quintuplo $B$, con tre piani tangenti fissi per la $b$, di cui uno passante per $c$, $\theta$ una $C^{(6)}$-base, sezione d'ana $\varphi^{(6)}$ con un cono quadrico con le generatrici $b$ é $c$.

Mediante una corrispondenza cremoniana data dal sistema omaloidico delle $\varphi^{(2)}$ per le rette $b, c$ e per un punto della $C^{(6)}$, il sistema di $\varphi^{(6)}$ si trasforma nel sistema lineare delle $\varphi^{(5)}$ con le relle-base b e e, rispettivamente tripla e semplice, incidenti in un punto quadruplo B, con ivi due piani tangenti fissi per la b, ed inoltre una $\mathrm{C}^{(5)}$-base, sezione d'una $\varphi^{(5)}$ con un piano generico per il punto B.

Per ottenere di questo sistema di $\varphi^{(5)}$ la rappresentazione analitica nel$l^{\prime} S_{3}(x, y, z)$ affine (n. 14), scegliamo come retta $c$ la retta impropria del 
piano $(x z)$, e la curvabase $C^{(5)}$ nel piano in equazione $y-z=0$. Si ottiene allora direttamente

$$
(y-z)\left(\lambda_{0} \bar{F}_{9} x+F_{3} y+G_{3}\right)+\lambda_{9}\left[\bar{F}_{2} x\left(F_{1} y+\bar{G}_{1}\right)+\bar{F}_{3} y^{3}+\bar{F}_{4} y+\bar{G}_{4}\right]=0 .
$$

V) $V_{3}^{(10)}$ dell' $S_{9}$ rappresentata nell' $S_{3}{ }^{*}$ dal sistema lineare delle $\varphi^{(6)}$ con una retta-base $b$ quadrupla, ana retta-base $c$ doppia, infinitamente vicina alla $b$ e complanare con essa, un punto quintuplo $B$ sulla $b$, con ivi tre piani tangenti fissi, ed inoltre una $C^{(6)}$.base, sezione $d^{\prime}$ una $\varphi^{(6)}$ con un cono quadrico di vertice $B$, colle generatrici $b$ e $c$.

Mediante la corrispondenza cremoniana data nell' $S_{3}^{*}$ dal sistema omaloidico delle $\varphi^{(2)}$ per $b, c$ e per un punto della $C^{(6)}$, il sistema di $\varphi^{(6)}$ si trasforma nel sistema delle $\varphi^{(5)}$ con un piano tangente fisso lungo una retta tripla $b$, su questa un punto quadruplo $\mathrm{B}$, con ivi due piani tangenti fissi per $\mathrm{b}$, ed inoltre una $\mathrm{C}^{(5)}$-base, sezione di una $\varphi^{(5)}$ con un piano generico per $\mathrm{B}$.

Per ottenere la rappresentazione analitica di.questo sistema lineare $\left|\varphi^{(5)}\right|$ nell' $S_{3}(x, y, z)$ affine (n. 14), scegliamo il punto $B$ nel punto improprio dell'asse $x$ e la $C^{(5)}$-base nel piano $(x z)$. Si ottiene allora direttamente

$$
\bar{F}_{1}\left(\lambda_{6} \bar{F}_{2} x y+F_{2} y^{2}\right)+F_{4} y+\lambda_{9}\left(\bar{F}_{2} \bar{G}_{2} x+\bar{F}_{5}\right)=0 .
$$

95. Una $V_{3}^{(11)}$ dell' $S_{10}$ (non cono), con un punto quadruplo $A$ è (se esiste) l'intersezione residua di un cono $M_{4}^{(7)}$ (il cui vertice è il punto $A$ oppure una retta $a$ passante per $A$ ) con una quadrica $Q_{\vartheta}$ (non cono di vertice $A$ ) che passa per tre spazi $S_{3}^{(1)}, S_{3}^{(2)}, S_{3}^{(3)}$ del cono $M_{4}^{(7)}$ (n. 10). Viceversa, una quadrica $Q_{9}$, che passi per i tre spazi $S_{3}{ }^{(1)}, S_{3}^{(2)}, S_{3}^{(3)}$, non è necessariamente un cono (quei tre spazi appartenendo o all' $S_{9}$ tangente alla $Q_{9}$ in $A$ oppure all' $S_{s}$ polare della retta $\alpha$ ); e se non passa di conseguenza per altri punti dell' $S_{10}$ (cioè se quei tre $S_{3}$ non hanno in comane con un medesimo $S_{h}(h>1)$ più di $\frac{h(h+2)}{2}-1$ punti generici), segnerà sugli $\infty^{1} S_{3}$ dell' $M_{4}^{(7)}$ le quadriche $Q$ di una $V_{3}^{(11)}$.

La $M_{3}^{(7)}$, sezione del cono $M_{4}^{(7)}$ con un $S_{3}{ }^{*}$ generico dell' $S_{10}$, può esseré rappresentata, nell' $S_{3}$. da un sistema lineare $\left({ }^{23}\right)$.

(23) Se la $M_{3}^{(7)}$ dell' $S_{3}$ non è un cono, essa si proietta biunivocamente da un suo piano in una $M_{3}^{(4)}$ dell' $S_{6}$, rappresentata nell' $S_{3}{ }^{*}$ dal sistema delle: I) $\varphi^{(2)}$ per una retta $b$; II) $\varphi^{(3)}$ con due rette-base $b$ e $c$, doppia e semplice, incidenti in un punto $B$, nel quale $\mathrm{i}$ due piani tangenti sono fissi; IIT) $\varphi^{(3)}$ con lungo una retta doppia $b$ un piano tangente fisso $e$ in un punto $B$ della $b$ fisso anche il secondo piano tangente; IV) $\varphi^{(4)}$ con lungo una retta tripla $b$ fissi i tre pianj tangenti. Sommando a questi sistemi un piano per $b$, si ottengono $i$ primi quattro sistemi rappresentativi di $M_{3}{ }^{(7)}$. Se la $M_{3}{ }^{(7)}$ è un $S_{0^{\text {mcono }}}$ (tenendo conto che una 
I) $\varphi^{(3)}$ con una retta doppia $b$;

II) $\varphi^{(4)}$ con due rette-base $b$ e $c$, rispettivamente tripla e semplice, incidenti in un punto $B$ in cai sono fissi due piani tangenti (di cui uno per c);

III) caso particolare del precedente, iu cui $b$ e $c$ sono infinitamente vicine;

IV) $\varphi^{(5)}$ con una retta quadrupla $b$ e lungo essa tre piani tangenti fissi;

V) $\varphi^{(4)}$ con una retta tripla $b$ in un suo punto $B$ fissi $i$ tre piani tangenti;

VI) $\varphi^{(5)}$ con una retta quadrupla $b$, lungo essa due piani tangenti fissi, $e$ in nn suo punto $B$ fissi anche gli altri due piani tangenti;

VII) $\varphi^{(6)}$ con una retta quintupla $b$, lungo essa quattro piani tangenti fissi, e in un suo punto $B$ fisso anche il quinto piano tangente.

In conseguenza (n. 21) otteniamo i seguenti quattro tipi di $V_{3}^{(11)}$ dell' $S_{10}$ poiehè (come preciseremo) non esistono i tipi IV e VII (ed il III si potră considerare come un caso particolare del II):

I) $V_{3}^{(1)}$ dell' $S_{10}$ rappresentata nell' $S_{3}^{*}$ dal sistema lineare delle $\varphi^{(3)}$ tangenti lungo una retta $b$; con la seguente rappresentazione analitica nel. I' $S_{3}(x, y, z)$ affine (n. 14).

$$
\lambda_{0}\left(\bar{F}_{1} x^{2}+\bar{G}_{1} x y+\bar{H}_{1} y^{2}\right)+F_{2} x+G_{2} y+F_{3}=0 .
$$

II) $V_{3}^{(11)}$ dell' $S_{10}$ rappresentata nell' $S_{3}{ }^{*}$ dal sistema lineare delle $\varphi^{(5)}$ con le rette-base $b$ e $c$, rispettivamente tripla e doppia, incidenti in un punto quadruplo $B$, nel quale due piani tangenti per $b$ (di cui uno anche per $c$ ) sono fissi; ed inoltre con una $C^{(3)}$-base, sezione d' una $\varphi^{(5)}$ con un piano generico per $c$.

-Mediante la corrispondenza cremoniana definita nell' $S_{3}^{*}$ dal sistema omaloidico delle $\varphi^{(2)}$ per $b, c$ e un punto della $C^{(3)}$ base, questo sistema di $\varphi^{(5)}$ si trasforma nel sistema delle $\varphi^{(4)}$ con le rette-base b e c, rispetlivamente doppia e semplice, incidenti in un punto triplo $\mathrm{B}$, tangenti lungo la retta $\mathrm{b}$, e in $\mathrm{B}$ fissi due piani tangenti (per b e di cui wno anche per o).

Per dare di questo sistema lineare di $\varphi^{(5)}$ la rappresentazione analitica nell' $S_{3}(x, y, z)$ affine (n. 14), supponiamo che la' retta $c$ sia rappresentata nel piano improprio dell' equazione $\bar{\lambda}_{0} y+\bar{\lambda}_{1} z=0$. Si ottiene allora

$$
\begin{gathered}
\lambda_{0}\left(\bar{F}_{1} x y+\bar{G}_{1} y^{z}\right)+\bar{F}_{1} F_{1} x+F_{2} y+F_{3}+ \\
+z\left(\lambda_{0} y+\bar{\lambda}_{1} z\right)\left(\lambda_{0} \bar{\lambda}_{2} \bar{F}_{1} x+\lambda_{0} \bar{\lambda}_{3} y z+\lambda_{10} z^{2}\right)=0 .
\end{gathered}
$$

superficie $F_{8}^{(7)}$ dell' $S_{8}$ (non cono è rappresentata nel piano da: $V$ ) sistema di $C^{(4)}$ con un punto triplo; $\nabla I)$ sistema di $C^{(5)}$ con un punto quadruplo ed ivi due tangenti fisse; VII) sistema di $C^{(6)}$ con an punto quintuplo ed ivi quattro tangenti fisse) si ricavano direttamente gli ultimi tre sistemi lineari di superficie rappresentativi di $M_{3}{ }^{(7)}$. 
Se teniamo conto che le rette $b$ e $c$ sono distinte, possiamo supporre nella (25) $\bar{\lambda}_{1}=0$. Allora la scrittura dell' equazione si semplifica nel seguente modo

$$
\lambda_{0}\left(\bar{F}_{1} \bar{G}_{1} x y+\bar{F}_{2} y^{2}\right)+\bar{F}_{1} F_{1} x+F_{3} y+G_{3}=0 .
$$

III) Il sistema lineare rappresentativo della $V_{3}^{(11)}$ è il caso particolare che si deduce dalla (25) ponendovi $\bar{\lambda}_{0}=0$. Si ottiene (ordinando opportunamente)

$$
\lambda_{0}\left(\bar{F}_{1} x y+\bar{G}_{1} y^{2}\right)+\bar{F}_{1}\left(F_{1}+\lambda_{0} z^{2}\right) x+\left(F_{2}+\lambda_{0} z^{3}\right) y+F_{4}=0
$$

si tratta cioè di un sistema lineare di $\varphi^{(1)}$ tangenti lungo una retta doppia b, con un piano tangente fisso, lungo il quale hanno un contatto del secondo ordine.

IV) In questo caso il nostro procedimento (n. 21) per costruire nell' $S_{3}{ }^{*}$ il sistema lineare rappresentativo dell' eventuale $V_{3}^{(11)}$ cade in difetto. Questo fatto si poteva facilmente prevedere. Infatti il cono $M_{4}^{\left({ }^{(4)}\right.}$, di vertice $A$, contiene un cono quadrico $K_{3}^{(2)}$, di vertice $A$, i cui $\infty^{1}$ piani di una schiera si distribuiscono sugli $\infty^{1} S_{3}$ dell' $M_{4}{ }^{(4)}$. Poichè la quadrica $Q_{9}$ contiene tre di questi piani, essa contiene tutto il cono $K_{3}^{(2)}$, cioè la sua $V_{3}^{(11)}$ sezione coll' $M_{4}^{(4)}$ si spezza in questo cono $K_{3}^{(2)}$ e in una $V_{3}^{(9)}$.

V) $V_{3}^{(11)}$ dell' $S_{10}$ rappresentata nell' $S_{3}{ }^{*}$ dal sistema lineare delle $\varphi^{(5)}$ per una retta tripla $\mathrm{b}$, con in un suo punto quadruplo $\mathrm{B}$ fissi tre piani tangenti per $\mathrm{b}$, e una $\mathrm{C}^{(5)}-b a s e$, sezione di una $\varphi^{(5)}$ con un piano generico per $\mathrm{B}$.

Come rappresentazione analitica di questo sistema lineare di $\varphi^{(5)}$ nell' $S_{3}(x, y, z)$ affine (n. 14), scelti il punto $B$ nel punto improprio dell'asse $x$ e la $C^{(5)}$-base nel piano $(x z)$, si ottiene

$$
\lambda_{8} \bar{F}_{3} x y+F_{9} y^{2}+F_{4} y+\lambda_{10}\left(\bar{F}_{3} \bar{F}_{8} x+\bar{F}_{5}\right)=0 .
$$

VI) Il nostro procedimento (n. 21) applicato direttāmente a questo caso, fornisce un sistema di $\varphi^{(\gamma)}$ con una retta-base $b$ quintupla, cui sono infinitamente vicine due rette-base doppie, $c, d$, incidenti la $b$ in un punto sestuplo $B$, in cui quattro piani tangenti sono fissi; ed inoltre con due coniche-base in due piani generici per $b$. Ma la dimensione di questo sistema lineare di $\varphi^{(7)}$ risulta 11, anzichè 10 . Il sistema di $\varphi^{(7)}$ ammetterà quindi un nuovo elemento base, che ora vogliamo trovare.

Perciò osserviamo che l' $S_{1}$-cono $M_{4}^{(7)}$ contiene un cono quadrico $K_{3}^{(2)}$, luogo di $\infty^{1}$ piani per l' $S_{1}$ vertice dell' $M_{4}^{(\eta)}$. Poichè la quadrica $Q_{9}$ (che segna la $V_{3}^{(4)}$ sull' $M_{4}^{(7)}$ ha in comune con $K_{3}^{(2)}$ tre piani, essa taglia ulterior. mente $K_{3}^{(2)}$ in un quarto piano. A questo piano corrisponde una giacitura per 
la retta-base $b$, cioè un nuovo piano tangente fisso che va imposto al sistema lineare $\left|\varphi^{(n)}\right|$.

Mediante la trasformazione cremoniana definita nell' $S_{3}^{*}$ dal sistema omaloidale delle $\varphi^{(3)}$ con la retta doppia $b$, le rette-base semplici $c$ e $d$, e un puntobase su ciascuna delle due coniche-base del sistema $\left|\varphi^{(7)}\right|$, questo sistema si trasforma nel sistema lineare delle $\varphi^{(5)}$ con tre piani tangenti fissi per una retta tripla $\mathrm{b}$, e lungo due di essi un contatto dè secondo ordine, e inoltre in un punto quartuplo $\mathrm{B}$ della b fissi due piani tangenti per $\mathrm{b}$.

Per dare di questo sistema di $\varphi^{(\hat{)})}$ la rappresentazione analitica nell' $S_{3}(x, y, z)$ affine (n. 14), scegliamo come punto $B$ il punto improprio dell'asse $x$ e come piani, lango i quali le $\varphi^{(5)}$ hanno un contatto del secondo ordine, il piano $(x y)$ e il piano improprio. Si ottiene allora direttamente

$$
\lambda_{0} \bar{F}_{1} z y^{2}+x\left(\lambda_{0} \bar{\lambda}_{0}+\lambda_{1} z+\lambda_{0} \bar{\lambda}_{2} z^{2}\right) \bar{F}_{2}+y\left(\lambda_{0} \lambda_{1}+z G_{2}+\lambda_{0} \bar{\lambda}_{3} z^{4}\right)+F_{5}=0 .
$$

VII) Anche in questo caso la costruzione (n. 21) del sistema lineare rappresentativo della $\mathrm{r}_{3}^{(11)}$ risulta impossibile. Ciò accade perchè gli $\infty^{4} S_{33}$ dell' $S_{1}$-cono $M_{4}^{(7)}$ tagliano uno spazio $S_{3}$ físso nei piani di un fascio. Poichè la $Q_{9}$ ha in comune con questo $S_{3}$ tre piani, essa contiene interamente questo $S_{3}$, cioè la $V_{3}^{(11)}$ sezione della $Q_{2}$ coll' $M_{4}^{(2)}$ si spezza in questo $S_{3}$ e in una $V_{3}^{(11)}$.

26. Una $V_{3}^{\left({ }^{2}\right)}$ dell' $S_{11}$, non cono, con un punto quadruplo $A$ è (se esiste) l'intersezione residna d'un cono $M_{4}^{(8)}$ dell' $S_{41}$ con una quadrica $Q_{10}$ (non cono di vertice $A$ ), che passa per quattro $S_{3}$ generatori dell' $M_{4}{ }^{(8)}$ (n. 10). Se $l^{\prime} M_{4}^{\left({ }^{(8)}\right.}$ è un $S_{0}$ rcono (di vertice $A$ ), quattro $S_{3}$ generatori appartengono all' $S_{14}$, quindi la $Q_{30}$ dovrebbe essere un cono (di vertice $A$ ), contro il supposto.

Rimane da considerare il caso che il vertice del cono $M_{4}{ }^{(8)}$ sia una retta $a$ (n. 11). Allora quattro $S_{3}$ generatori appartengono all' $S_{9}$, spazio polare rispetto alla $Q_{10}$ della retta $a$. La superficie-sezione $F_{\mathrm{s}}^{\left({ }^{(8)}\right.}$ dell' $S_{1}$-cono $M_{4}^{(8)}$ con un $S_{9}$ generico dell' $S_{11}$, puo avere una direttrice minima di ordine 1,2.3 o infinite direttrici minime d'ordine 4 . Il caso che la direttrice minima sia d'ordine 1 va escluso. Infatti, l' $S_{3}$ determinato dall' $S_{1}$, vertice del cono, e dalla direttrice minima è segato dagli $S_{3}$ generatori in piani, quindi la $Q_{10}$, avendo colI' $S_{3}$ quattro piani in comune, conterrebbe questo $S_{3}$, cioè la $V_{3}{ }^{(12)}$, sezione della $Q_{10}$ coll' $M_{4}^{(8)}$, si spezzerebbe.

La $M_{3}^{(8)}$, sezione dell' $S_{1}$-cono $M_{4}^{(8)}$ con un $S_{10}{ }^{*}$ generico dell' $S_{11}$, è rappresentata nell' $S_{3}^{*}$ da un sistema lineare di $\left({ }^{24}\right)$

$\left.{ }^{24}\right)$ Basta tener presente che la $F_{2}^{(8)}$, a seconda che $I^{\prime}$ ordine della direttrice minima è 2, 4 o 3 , è rappresentata nel piano da un sistema lineare di I) $C^{(6)}$ con 3 tangenti fisse in un punto quintuplo; II) $C^{(5)}$ con un punto-base quadruplo e uno semplice; III) $C^{(5)}$ con una tangente fissa in un punto-base quadruplo. 
I) $\rho^{(0)}$ con una retta-base $b$ quintupla, lungo essa tre piani tangenti fissi, e in un suo punto $B$ fissi tutti i cinque piani tangenti;

II) $\varphi^{(5)}$ con due rette-base, $b$ e $c$, rispettivamente quadrupla e semplice, incidenti in un punto $B$, nel quale sono fissi i quattro piani tangenti;

III) $\varphi^{(5)}$ con una retta quadrupla $b$, lungo essa un piano tangente fisso, e in un suo punto $B$ fissi i quattro piani tangenti.

In conseguenza si hanno (n. 21) i seguenti tipi di $V_{3}^{(1 *)}$ dell' $S_{11}$ :

I) $V_{3}^{(12)}$ dell' $S_{i 1}$ rappresentata nell' $S_{3}{ }^{*}$ dal sistema lineare delle $\varphi^{(8)}$ con una retta-base $b$ sestupla, tre rette-base $c, d, e$ doppie, infinitamente vicine alla $b$ e ad essa incidenti in un punto sestuplo $B$, con ivi cinque piani tangenti fissi per $b$; ed inoltre due coniche-base, appartenenti a due piani per la retta 6 . Mediante la corrispondenza cremoniana definita nell' $S_{3}^{*}$ dal sistema lineare delle $\varphi^{(4)}$ colla retta tripla $b$, le rette semplici $c, d$, e, un puntorbase su ciascuna delle due coniche-base del sistema $\left|\varphi^{(8)}\right|$ e inoltre un terzo puntobase generico, questo sistema di $\varphi^{(8)}$ si trasforma nel sistema lineare di $f^{(6)}$ con una retta quadrupla $\mathrm{b}$, tre rette-base $\mathrm{c}, \mathrm{d}$, e infinitamente vicine alta $\mathrm{b}$, di cui c doppia e con un contatto del secondo ordine lungo d ed e, incidenti la b in un punto quintuplo $\mathrm{B}$, con ivi due piani tangenti fissi (oltre il piano bc).

Per dare di questo sistema lineare di $\varphi^{(6)}$ la rappresentazione analitica nell' $S_{3}(x, y, z)$ affine (n. 14), scegliamo come punto $B$ il punto improprio dell'asse $x$, la retta $c$ nel piano di equazione $\bar{F}_{1}=0$, e le rette $d$ ed $e$ rispetti. vamente nel piano $(x y)$ e nel piano improprio. Si trova allora direttamente

$\lambda_{4} \bar{F}_{1}^{2} z y^{3}+\bar{F}_{1}\left[x\left(\lambda_{0} \bar{\lambda}_{0}+\lambda_{1} z+\lambda_{0} \bar{\lambda}_{2} z^{2}\right) \overline{F_{2}}+y\left(\lambda_{0} \bar{\lambda}_{1}+z G_{2}+\lambda_{0} \bar{\lambda}_{3} z^{4}\right)+F_{6}=0\right.$.

II) $V_{3}^{\left({ }^{(2)}\right)}$ dell' $S_{11}$ rappresentata nell' $S_{3}^{*}$ dal sistema lineare delle $\varphi^{(0)}$ con due rette-base $b, c$, rispettivamente quadrupla e doppia, incidenti in un punto quintuplo $B$, con ivi quattro piani tangenti fissi per $b$; e una $C^{(4)}$.base, sezione di una $\varphi^{(6)}$ con un piano generico per la retta $c$. Mediante la corrispondenza cremoniana definita dal sistema omaloidico delle $\varphi^{(2)}$ per le rette-base $b, c$ e un punto-base sulla $O^{(4)}$, il sistema di $\varphi^{(6)}$ si trasforma nel sistema lineare di $\varphi^{(5)}$ coi piani tangenti fissi nei punti di una retta tripla b e una retta-base semplice $\mathrm{c}$, incidente la $\mathrm{b}$ in un punto quintuplo $\mathrm{B}$, in cui tre piani tangenti per b sono fissi.

Nell' $S_{3}(x, y, z)$ affine (n. 14), scegliamo come retta $c$ la retta impropria del piano $(x z)$. Otteniamo allora come rappresentazipne analitica del sistema lineare $d \mathbf{i} \varphi^{(5)}$

$$
\lambda_{n}\left(\bar{F}_{3} x y+\bar{G}_{3} y^{2}\right)+\lambda_{1} \bar{F}_{3} x+G_{4} y+F_{4}=0 .
$$

III) Se al sistema lineare rappresentativo dell' $M_{3}{ }^{\left({ }^{8}\right)}$ di questo tipo, 
applichiamo diretiamente il procedimento del n. 21 (senza le dovute avver. tenze per l'intorno della retta $b$ ), otteniamo un sistema lineare di $\varphi^{(6)}$, con nna retta quadrupla $b$, una retta doppia $c$, complanare con $b$ e ad essa infinitamente vicina, in un punto quintuplo $B$ della $b$ quattro piani tangenti fissi per la $b$; ed inoltre con una conicabase, sezione di una $\varphi^{(6)}$ con un piano generico per la retta $b$. Dalla considerazione di questo sistema risulta che la sua dimensione è 13 , anzichè 11. Andiamo dunque a ricercare gli elementi. base insinuati nell' intorno della $b$ !

Ricordiamo che il cono $M_{4}^{\left({ }^{(8)}\right.}$ è generato dagli $\infty^{1} S_{3}$ che da una retta $a$ proiettano una rigata $F_{2}^{(8)}$ con una cubica direttrice $C^{(3)}$. Quindi $M_{4}^{(8)}$ contiene il cono cubico $K_{3}{ }^{(3)}$, generato dagli $\infty^{1}$ piani che da una retta $a$ proiettano i punti della $C^{(3)}$. La quadrica $Q_{10}$ (che segna sul cono $M_{4}^{(8)}$ la $V_{3}^{(12)}$ ) passa per quattro piani del cono $K_{3}{ }^{(3)}$ e quindi (poichè la $Q_{4}$ sezione di $Q_{10}$ coll' $S_{5}$ d'appartenenza di $K_{3}{ }^{(3)}$ è un cono di vertice $a$ ) sega ulteriormente questo cono in altri due piani. Il cono $K_{2}^{(11)}$ delle rette della $V_{3}^{(1+)}$ che passano per il punto $A$ (n. 21), si spezza dunque in questo caso in due piani per la retta $b$ ed in un

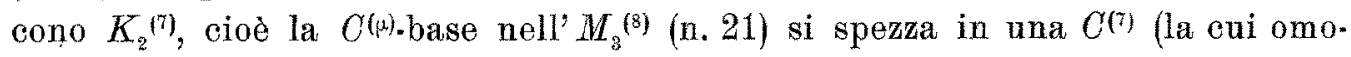
loga nell' $S_{3}^{*}$ è la conica-base del sistema lineare di $\varphi^{(6)}$ ) ed in due generatrici del cono $K_{2}{ }^{(3)}$ (sezione del cono $K_{3}{ }^{\left({ }^{3}\right)}$ coll' $S_{10}{ }^{*}$ dell' $M_{3}{ }^{(8)}$ ).

Nella rappresentazione dell' $M_{3}{ }^{\left({ }^{8}\right)}$ nell' $S_{3}{ }^{*}$, alle generatrici del cono $K_{2}{ }^{(3)}$ corrispondono le giaciture per la retta $b$. Nel sistema lineare di $\varphi^{(6)}$, precedentemente descritto, dobbiamo dunque introdurre come nuovi elementi-base due piani tangenti fissi per la retta $b$.

Mediante la corrispondenza cremoniana definita nell' $S_{3}^{*}$ dal sistema oma* loidico delle quadriche che hanno lungo la retta-base $b$ il piano tangente $(b c)$ fisso e un punto-base sulla conica-base del sistema lineare $\left|\varphi^{(6)}\right|$, questi si trasforma nel sistema lineare di $\varphi^{(5)}$ con tre piani tangenti fissi lungo una retta tripla $b$, lungo uno di essi un contatto del secondo ordine, e in un punto quadruplo $\mathrm{B}$ della b fissi tre piani tangenti.

Nell' $S_{3}(x, y, z)$ affine (n. 14) scegliamo come punto $B$ il panto improprio dell' asse $x$, come piano tangente lungo il quale le $\varphi^{(5)}$ hanno in contatto del secondo ordine il piano $(x y)$. Otteniamo allora per la relativa rappresentazione analitica

$$
\lambda_{0} \bar{F}_{2} z y^{2}+x \bar{G}_{3}\left(\lambda_{0} \bar{\lambda}_{0}+\lambda_{1} z\right)+y\left(\lambda_{0} \bar{\lambda}_{1}+z F_{3}\right)+F_{5}=0 .
$$

27. Come conclusione a questo paragrafo verifichiamo che una $\mathrm{V}_{3}{ }^{(13)}$ del$l ' \mathrm{~S}_{12}$, con un punto quadruplo $\mathrm{A}$, è necessariamente un cono.

Infatti, sul cono $M_{4}^{\left({ }^{\circ}\right)}$, il cui vertice è il punto $A$ o una retta $a$ passante per $A$, la $V_{3}^{(13)}$ è segata da una quadrica $Q_{11}$, passante per cinque spazi $S_{3}$ 
dell' $M_{4}{ }^{(9)}$, (n. 10). Se il vertice del cono $M_{4}{ }^{\left({ }^{9}\right)}$ è il punto $A$, allora lo spazio d'appartenenza dei cinque $S_{3}$ è l' $S_{12}$ stesso, cioè la $Q_{11}$ è un cono di vertice $A$. Di conseguenza anche la $V_{3}^{(13)}$ è un cono di vertice $A$.

Se il vertice del cono $M_{4}{ }^{(9)}$ è la retta $a$, allora $i$ cinque $S_{3}$ appartengono ad un $S_{11}$. La $Q_{11}$, avendo l' $S_{14}$-tangente fisso lungo la retta $a$, è un cono, col vertice sulla retta $A$, cioè la $V_{n}^{\left({ }^{(3)}\right)}$ stessa è un cono.

Queste considerazioni valgono a più forte ragione per una $V_{3}^{(r+1)}$ del. l' $S_{r}$, con $r>12$.

\section{§ 3. $V_{3}^{\left({ }^{(8)}\right.}$ dell' $S_{7}$, priva di punti quadrupli, per eui $l^{9} M_{4}^{\left({ }^{4}\right)}$ è un $S_{0}$-cono.}

28. In questo caso la $V_{3}{ }^{(8)}$ è l'intersezione completa del cono $M_{4}^{\left({ }^{(4)}\right.}$ con una quadrica generica $Q_{6}$ dell' $S_{7}$ (n. 10). L'ipotesi che il vertice del cono $M_{4}{ }^{(4)}$ possa essere una retta va $q u i$ esclusa, poichè i punti comuni a questa retta e all' iperquadrica $Q_{6}$ sarebbero quadrupli per la $V_{3}^{\left({ }^{(8)}\right.}$ (n. 22).

La $M_{3}{ }^{\left({ }^{*}\right)}$ sezione dell' $M_{4}^{\left({ }^{(1)}\right.}$ con un $S_{6}{ }^{*}$ generico dell' $S_{7}$ si proietta biunivocamente da un suo piano generico sopra un $S_{3}^{*}$, le superficie sezioni dell' $M_{3}{ }^{\left({ }^{*}\right)}$ risultando ivi rappresentate dalle quadriche per una retta $b$. Alla retta $b$ corrisponde viceversa una quadrica $Q_{2}$ dell' $M_{3}{ }^{(4)}$, alla quale i piani dell' $M_{s}^{(+)}$si appoggiano lungo le generatrici di una sčhiera. Cioè nel cono $M_{4}^{\left({ }^{()}\right.}$ (di vertice $A$ ) esiste un cono quadrico $K_{3}{ }^{\left({ }^{2}\right)}$ (di vertice $A$ ), segato nei piani d' una schiera dagli $\infty^{1} S_{3}$ del cono $M_{4}^{(4)}$.

L'iperquadrica $Q_{6}$ segna sul cono $K_{3}{ }^{(2)}$ una superficie $F_{2}{ }^{(4)}$, a curve-sezioni ellittiche, sulla quale, in corrispondenza alle dne schiere di piani del cono $K_{3}{ }^{\left({ }^{2)} \text {, }\right.}$ vi sono due fasci di coniche. Le coniche d' un fascio sono le sezioni delle $\infty^{1}$ quadriche $Q$ della $V_{3}{ }^{\left({ }^{8}\right)}$ col cono $K_{3}{ }^{\left({ }^{\prime}\right)}$ (cioè coll' $S_{4}$ di questo cono). Una retta $a$, componente d' una conica riducibile dell' altra schiera $\left({ }^{25}\right)$, è dunque una retta unisecante le quadriche del fascio $|\mathrm{Q}|$.

Sia $B_{1}$ il punto d'incontro della retta $a$ coll' $S_{6}^{*}$ dell' $S_{7}, \beta_{1}$ il piano dell' $\dot{M}_{3}^{\left({ }^{(4)}\right.}$ dell' $S_{6}^{*}$ passante per $B_{1}, C^{(4)}$ la curva-sezione dell' $F_{\varepsilon}^{\left({ }^{(4)}\right.}$ coll' $S_{6}^{*}$, passante per il punto $B_{1}$ e appartenente alla quadrica direttrice $Q_{2}$ dell' $M_{3}{ }^{(4)}$.

La generica quadrica $Q$ della $V_{3}{ }^{(8)}$ ha in comune colla retta $\alpha$ un punto $A$. Proiettando la $Q$ da $A$ su $\beta$ (piano dell' $M_{3}{ }^{\left({ }^{4}\right)}$ traceia sull' $S_{6}^{*}$ dell' $S_{3}$ d'appartenenza della $Q$ ), al variare della $Q$ si ottiene una rappresentazione birazionale della $V_{3}{ }^{\left({ }^{8}\right)}$ sull $M_{3}{ }^{(4)}$. Il sistema lineare di superficie $j \Phi \mid$, rappresentativo della $V_{3}^{\left({ }^{(8)}\right.}$ sull $M_{3}^{\left({ }^{(4)}\right.}$ ha come elementi-base il punto doppio $B_{1}$ (al quale cor-

(25) L' $F_{2}^{(4)}$, superficie di SEGRE, è rappresentata nel piano dalle cubiche per 5 punti. Quindi in un fascio di coniche vi sono 4 coniche degeneri. 
risponde la quadrica $Q$ per questo punto) e la curva $C(\mu)$, traccia sull' $S_{6} *$ della rigata formata dalle generatrici delle quadriche $Q$ che passano per $\mathrm{i}$ punti $A$ della retta $a$. Ad un punto della conica, sezione del piano $\beta_{t}$ (per $B_{t}$ ) colla $V_{a}{ }^{(8)}$, corrisponde la retta che lo proietta da $B_{4}$; cioè le superficie $\Phi$ hanno in comune col piano $\beta_{1}$ coppie di rette per $B_{1}$.

Una particolare superficie del sistema $|\Phi|$ è la $F^{\left({ }^{(}\right)}$, sezione della $V_{3}{ }^{\left({ }^{8}\right)}$ coll' $S_{6}^{*}$, contata assieme al piano $\beta_{1}$. Dunque le $\Phi$ sono del nono ordine. Alle superficie-sezioni della $V_{3}{ }^{(8)}$, cogli $S_{6}$ per la retta $a$, corrispondono sulla $M_{3}{ }^{(4)}$ le superficie-sezioni cogli $S_{5}{ }^{*}$ per il punto $B_{1}$, contate assieme alla rigata $\psi^{(0)}$, imagine della retta $a$ (la $\psi^{(5)}$ essendo il luogo delle tracce sull' $S_{6}{ }^{*}$ dei piani tangenti alle $Q$ nei punti $A$ della retta $a$ ).

Questa rigata $\psi^{(5)}$ e la $F^{(8)}$ sono tangenti nel punto $B_{1}$. Infatti la varietà a tre dimensioni generata dai piani tangenti alle $Q$ nei punti della retta $a$ la $V_{3}^{(8)}$ hanno in un punto generico $A$ della retta $a$ (in particolare in $B_{1}$ ) lo stesso $S_{3}$-tangente, individuato dalla retta $a$ e dalle due rette della $Q$ che escono da $A$. Quindi le superficie $\psi^{(5)}$ e $F^{(8)}$, che sono le rispettive sezioni di quelle varietà coll' $S_{3}^{*}$, avranno in $B_{4}$ lo stesso piano tangente.

Se l' $S_{6}$ dell' $S_{7}$ per la retta $a$ passa in particolare per la $F_{2}{ }^{(4)}$, il corrispondente $S_{5}^{*}$ dell' $S_{6}{ }^{*}$ sega l' $M_{3}{ }^{(4)}$ in due piani e nella quadrica $Q_{\text {. }}$ (imagine

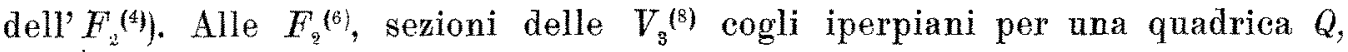
corrispondono sull' $M_{3}^{\left({ }^{(4)}\right.}$ superficie dell' ottavo ordine $\Phi^{(8)}$ col punto doppio $B_{1}$. La curva-base $C^{(m)}$ è l'intersezione completa della $\psi^{(5)}$ con una $\Phi^{(8)}$.

$L^{\prime} M_{3}{ }^{(4)}$ si proietta biunivocamente da un suo piano generico $\beta$ sopra un $S_{3}^{*}$, e al suo fascio di piani $|\beta|$ corrisponde nell' $S_{3}^{*}$ un fascio di piani, il cui asse sia la retta $b$. Le $\Phi^{(0)}$, segate dai piani $\beta$ in coniche, si proiettano in superficie $\varphi^{(5)}$, del quinto ordine (n. 12), colla retta tripla $b$ e il punto doppio $B$, proiezione di $B_{1}$. La $\psi^{(5)}$, che ha in comune col piano $\beta$ una retta, si proietta in una $\varphi^{(3)}$, colla retta doppia $b$ e il punto semplice $B$.

In conclusione, la $V_{3}{ }^{\left({ }^{*}\right)} \dot{e}$ rappresentata nell' $S_{3}{ }^{*}$ dal sistema lineare delle $\varphi^{(5)}$, con una retta-base tripla b e un punto-base doppio $\mathrm{B}$, e una $\mathrm{O}^{(8)}$ base, sezione d' una $\varphi^{(4)}$, colla retta doppia b e il punto doppio $\mathrm{B}$, e di una $\varphi^{(3)}$, colla retta doppia b e il punto semplice $\mathrm{B}$,

Come rappresentazione analitica di 'questo sistema di $\varphi^{(5)}$ nell' $S_{3}(x, y, z)$ affine (n. 14), scelto il punto doppio $B$ nell' origine, si ottiene

$$
\begin{gathered}
\left(\bar{F}_{2} x+\bar{G}_{2} y+\bar{H}_{2} z\right)\left(F_{1} x+G_{1} y+H_{1} z\right)+ \\
+\left[\bar{L}_{2} x^{2}+\bar{M}_{2} y^{2}+\bar{N}_{2} z^{2}+\left(\bar{O}_{2} x+P_{2} y+\bar{Q}_{2} z\right) z\right) L_{1}=0 .
\end{gathered}
$$




\section{\$ 4. La $V_{3}^{(7)}$ dell' $S_{6}$.}

29. La $V_{3}{ }^{(7)}$ a curve-sezioni iperellittiche di genere $\pi=3$ si trova su una $M_{4}^{\left({ }^{3}\right)}$ dell' $S_{6}$, luogo di $\infty^{1}$ spazi $S_{3}$, cono di vertice. $S_{n}, S_{1}$ oppure $S_{2}$. Vogliamo provare che la $\nabla_{3}{ }^{(7)}$ è l'intersezione residua del cono $\mathrm{M}_{4}{ }^{(3)}$ con un'ipersuperficie $\mathrm{M}_{5}{ }^{(3)}$ che passa per un cono quadrico $\mathrm{M}_{3}{ }^{\left({ }^{2}\right)}$ contenuto nell' $\mathrm{M}_{4}{ }^{\left({ }^{3}\right)}$.

Infatti le $M_{5}^{\left({ }^{(3)}\right.}$ dell' $S_{6}$ segnano sopra la $V_{3}^{\left({ }^{7}\right)}$ dell' $S_{6}$ un sistema lineare di superficie $\Phi$ di dimensione $\delta$, le quali segnano sopra una superficie-sezione $F_{2}{ }^{(7)}$ della $V_{3}{ }^{(7)}$ un sistema lineare di eurve $\Gamma$ (eventualmente non completo), di dimensione $\delta_{1}$. Le superficie $\Phi$ che contengono parzialmente la $F_{2}^{(7)}$ si spezzano in questa superficie $e$ in un sistema lineare $\{\psi\}$ che contiene totalmente (o coincide) col sistema di superficie determinato sulla $V_{3}^{(i)}$ dal sistema delle iperquadriche dell' $S_{6}$. Poichè le quadriche linearmente indipendenti dell' $S_{6}$ che passano per il cono $M_{4}{ }^{(3)}$ sono 3 , la dimensione $\delta_{2}$ del sistema $|\psi|$ è

$$
\delta_{2} \geq\left(\begin{array}{c}
6+2 \\
2
\end{array}\right)-1-3=24
$$

Il sistema di curve $\Gamma$ è contenuto totalmente nel sistema triplo del sistema delle curve-sezioni della $F_{z}{ }^{(7)}$. Quindi per il suo grado $n^{\prime}$ e il suo genere $\pi^{\prime}$ si ha

$$
n^{\prime}=3^{2} \cdot 7=63, \quad \pi^{\prime}=3 \cdot 3+3 \cdot 7-2=28,
$$

cioè la dimensione della serie-caratteristica completa del sistema $|\Gamma|$ è $n^{\prime}-\pi^{\prime}=63-28=35$. Dunque la dimensione del sistema di curve $\Gamma$ è $\delta_{1} \leq 36$. Quindi per la dimensione del sistema lineare delle $\Phi$ si ha

$$
\delta=\delta_{1}+\delta_{2}+1 \leq 36+24+1=61 \text {. }
$$

Poichè il sistema lineare delle ipersuperficie cubiche dell' $S_{6}$ ha la dimensione $D=\left(\begin{array}{c}6+3 \\ 3\end{array}\right)-1=84$, il numero delle ipersuperficie cubiche linear. mente indipendenti che contengono la $V_{3}{ }^{(7)}$ è

$$
D-\delta \geq 84-61=23 \text {. }
$$

Di queste 20 si possono considerare passanti per il cono $M_{4}^{(3)},\left({ }^{26}\right)$, quindi vi sono (almeno) $23-20=3$ ipersuperficie cubiche linearmente indipendenti che contengano la $V_{3}^{\left({ }^{(7)}\right.}$ senza contenere il cono $M_{4}^{\left({ }^{3}\right)}$. Una di esse sega dunque il cono $M_{4}^{\left({ }^{(2)}\right.}$ secondo la $V_{3}{ }^{(1)}$, ed ulteriormente in una.quadrica a

(26) loc. cit. (17), pag. 263. 
tre dimensioni, $M_{3}{ }^{(2)}$, la quale è quindi un cono il cui vertice appartiene al vertice del cono $M_{4}^{\left({ }^{(3)}\right.}$.

30. Sia $A$ il vertice dell' $S_{0}-$ cono $M_{3}{ }^{(2)}$ (o un punto del vertice dell' $S_{1}-$ o $S_{2}$-cono $\left.M_{3}{ }^{(2)}\right)$. Questo punto $A$ è quindi doppio per la $M_{5}^{(3)}$ e quadruplo per la $V_{3}{ }^{(7)}$. Dal punto $A$ la $V_{3}^{(7)}$ si proietta pereio biunivocamente sulla $M_{3}^{(3)}$ sezione del cono $M_{4}{ }^{(3)}$ con un $S_{5}{ }^{*}$ generico dell' $S_{6}$. Sulla $M_{3}{ }^{\left({ }^{3}\right)}$ la $V_{3}{ }^{(7)}$ è rappresentata da un sistema lineare di superficie $|\Phi|$.

Una particolare superficie del sistema $\Phi$ e la $F^{(\eta)}$ sezione della $V_{3}^{(7)}$ coll' $S_{4}{ }^{*}$. Essa è segata sulla $M_{3}{ }^{(3)}$ dalla $M_{4}{ }^{(3)}$ dell' $S_{5}{ }^{*}$, sezione con esso della $M_{5}{ }^{\left({ }^{3}\right)}$; passante per la quadrica $M_{2}{ }^{\left({ }^{3}\right)}$ della $M_{3}{ }^{\left({ }^{3}\right)}$, sezione dell' $S_{4}$ col cono quadrico $M_{3}{ }^{(2)}$. Quindi la $F^{(5)}$ appartiene al sistema lineare di superficie $|\Phi|$ segate sulla $M_{3}{ }^{\left({ }^{3}\right)}$ dalle $M_{4}{ }^{(3)}$ dell' $S_{5}$ che passano per la quadrica $M_{2}{ }^{\left({ }^{2}\right)}$, ed hanno come curva-base $C^{(\mu)}$ la traccia sull' $S_{5}^{*}$ del cono $K_{2}$ generato dalle generatrici della $Q$ della $V_{3}^{(7)}$ passanti per il punto $A$.

Il cono $K_{2}$ è la sezione della $V_{3}{ }^{(7)}$ col cono $K_{5}{ }^{\left({ }^{2}\right)}$ delle tangenti alla $M_{5}{ }^{(3)}$

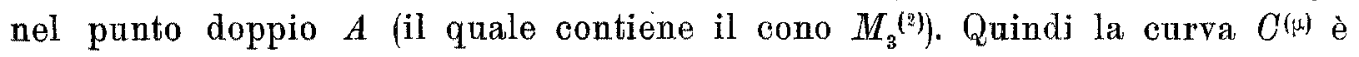
la sezione della $F^{(7)}$ con un'iperquadrica dell' $S_{5}^{*}$ che passa per la quadrica $M_{2}{ }^{(2)}$, e sega ulteriormente la $M_{3}{ }^{\left({ }^{(3)}\right.}$ in una $\Phi^{(4)}$.

31. Se la $M_{3}^{\left({ }^{3}\right)}$ dell' $S_{5}^{*}$ non è un cono, essa è rappresentata nell' $S_{3}^{*}$

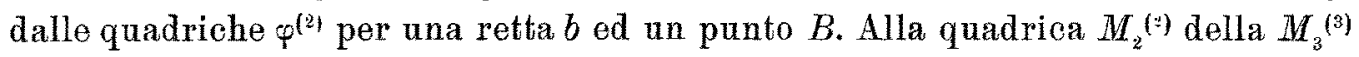
corrisponde un piano $\gamma$ dell' $S_{3}^{*}$ per il punto $B$. Il sistema delle sezioni della $M_{3}^{\left({ }^{(3)}\right.}$ colle $M_{4}^{\left({ }^{(3)}\right.}$ dell' $S_{5}{ }^{*}$ è quindi rappresentato da superficie del $6^{\circ}$ ordine, $\varphi^{(6)}$, colla retta tripla $b$ e il punto triplo $B$. Il sistema delle superficiesezioni della $M_{3}{ }^{(3)}$ colle $M_{1}{ }^{(3)}$ per la $M_{2}{ }^{\left({ }^{(2)}\right.}$ è il sistema residuo di $\varphi^{(5)}$ rispetto al piano $\gamma$, ćioè $\varphi^{(5)}$ con la retta tripla $b$ e il punto doppio $B$. La superficie dell' $S_{3}^{*}$ corrispondente della $\Phi^{(4)} \dot{e}$ analogamente una $\varphi^{(3)}$ con la retta doppia $b$ e il punto semplice $B$.

In conclusione la $V_{3}{ }^{(7)}$ è rappresentata nell' $S_{3}{ }^{*}$ dal sistema lineare nelle $p^{(5)}$ con una retta-base tripla $\mathrm{b}$, un punto-base doppio $\mathrm{B}$ e una $\mathrm{C}^{(9)}$-base, sezione di una $\varphi^{(5)}$ con una $\varphi^{(3)}$ con la retta-base doppia b e il punto-base semplice $\mathrm{B}$.

La rappresentazione analitica di questo sistema lineare nell' $S_{3}(x, y, z)$ affine (n. 14), assunto il punto doppio $B$ nell' origine, è

$$
\begin{gathered}
\left(\bar{F}_{2} x+\bar{G}_{2} y+\bar{H}_{2} z\right)\left(F_{1} x+G_{1} y+H_{1} z\right)+ \\
+\lambda_{6}\left[\bar{F}_{3} x^{2}+\bar{G}_{3} x y+\bar{H}_{3} y^{2}+z\left(\bar{L}_{3} x+\bar{M}_{3} y+\bar{N}_{3} z\right)\right]=0
\end{gathered}
$$


32. Se la $M_{3}{ }^{\left({ }^{3}\right)}$ dell' $S_{5}^{*}$ è un $S_{0}$-cono, essa viene rappresentata nell' $S_{3}{ }^{*}$ dal sistema lineare delle $\varphi^{(9)}$ per una retta $b$, col piano tangente $\beta$ fisso in un punto $B$ della $b$. Quindi (procedendo come al n. 21), la $V_{3}^{(7)}$ è rappresentata nell' $S_{s}^{*}$ dal sistema lineare delle $\varphi^{(5)}$ con una retta tripla b, un piano tangente tacnodale $\beta$ in un punto $\mathrm{B}$ di $\mathrm{b}$, e una $\mathrm{C}^{(9)}-b a s e$, sezione d' una $\varphi^{(5)}$ con una $\varphi^{(3)}$, colla retta doppia b e il piano tangente $\beta$.

Per dare di questo sistema di $\varphi^{(5)}$ la rappresentazione analitica nell' $S_{3}(x, y, z)$ affine (n. 14), scegliamo il punto $B$ nel punto improprio dell'asse $x$. Si ottiene allora

$$
\begin{gathered}
\left(\bar{F}_{1} G_{1} x+\bar{F}_{2} y+\bar{F}_{3}\right)\left(\lambda_{0} \bar{F}_{1} x+F_{1} y+F_{2}\right)+ \\
+\lambda_{6}\left[\bar{F}_{1}^{2} \bar{H}_{1} x+\bar{F}_{1}\left(\bar{G}_{2} y+\bar{G}_{3}\right) x+\bar{H}_{3} y^{2}+\bar{F}_{4} y+\bar{F}_{5}\right]=0 .
\end{gathered}
$$

Se la $M_{3}{ }^{(3)}$ dell' $S_{5}{ }^{*}$ è un $S_{1}$-cono, la $M_{4}^{\left({ }^{(3)}\right.}$ dell' $S_{6}$ è un $S_{z}$-cono. Il piano $\alpha$, vertice di questo cono, è allora quadruplo per la $V_{3}{ }^{(\mathrm{i})}$; e l'ulteriore intersezione col cono $M_{4}^{(3)}$ della $M_{5}^{(3)}$ per la $V_{3}{ }^{(n)}$ si spezza in due $S_{3}$ di questo cono.

L' $M_{3}{ }^{\left({ }^{3}\right)}$ è in questo caso rappresentata nell' $S_{3}{ }^{*}$ dal sistema lineare delle superficie $\varphi^{(3)}$ con due piani tangenti fissi lungo una retta doppia $b$, cioè due rette-base semplici, $c$ e $d$, complanari con $b$ e ad essa infinitamente vicine.

Quindi (n. 21), la $V_{3}^{(7)}$ è rappresentata dal sistema lineare delle $\varphi^{(7)}$ con la retta quintupla $b$, le rette doppie $c$ e $d$, ed una $C^{(9)}$-base, sezione d' una $\varphi^{(7)}$ con una $\varphi^{(t)}$ con la retta tripla $b$ e le rette semplici $c$ e $d$.

Mediante la trasformazione cremoniana data nell' $S_{3}^{*}$ dal sistema lineare delle $\varphi^{(3)}$, colla retta doppia $b$, le rette semplici $c$, $d$ e due punti-base semplici sulla $C^{9}$, il sistema lineare di $\varphi^{(7)}$ si trasforma nel sistema lineare delle $\varphi^{(5)}$, con due piani tangenti fissi lungo una retta tripla $\mathrm{b}$, ed una $\mathrm{C}^{(7)}$-base, sezione di una $\varphi^{(5)}$ con una $\varphi^{(2)}$ per b.

Nell' $S_{3}(x, y, z)$ affine (n. 14), questo sistema di $\varphi^{(5)}$ è rappresentato direttamente da

$$
\begin{gathered}
{\left[\bar{F}_{1} x+\bar{G}_{1} y+\bar{F}_{2}\right]\left[G_{2}\left(\lambda_{0} x+\lambda_{1} y\right)+F_{3}\right]+} \\
+\lambda_{6}\left[\bar{G}_{2}\left(\bar{H}_{1} x^{2}+\bar{L}_{4} x y+\bar{M}_{1} y^{2}\right)+\bar{F}_{4} x+\bar{G}_{4} y+\bar{F}_{5}\right]=0 .
\end{gathered}
$$

\section{$\S 5$. La $V_{3}^{(6)}$ dell' $S_{5}$.}

33. Gli $S_{3}$ delle quadriche $Q$ formano un cono quadrico $M_{4}^{\left({ }^{(2)}\right.}$ (n. 11), il cui vertice può essere una retta oppure un piano $\alpha$. 
Dimostriamo che la $\mathrm{V}_{3}^{\left({ }^{(b)}\right.}$ è l'intersezione residua del cono $\mathrm{M}_{4}{ }^{(2)}$ con un' ipersuperficie $\mathbf{M}_{4}{ }^{(4)}$, del quarto ordine, che passa per due $\mathrm{S}_{3}$ della $M_{4}^{\left({ }^{(2)}\right.},\left({ }^{(7)}\right)$.

Infatti le $M_{4}^{\left({ }^{(}\right)}$dell' $S_{5}$ segnano sopra la $V_{3}^{\left({ }^{(}\right)}$un sistema lineare di superficie $\Phi$ di dimensione $\delta$, le quali segnano sopra una superficie-sezione $F_{2}{ }^{(6)}$ della $V_{3}{ }^{(6)}$ un sistema lineare di curve $\Gamma$ (eventualmente non completo), di dimensione $\delta_{t}$. Le superficie $\Phi$ che contengono parzialmente la $F_{2}^{\left({ }^{6}\right)}$ si spezzano in questa superficie e in una superficie del sistema lineare $\{\downarrow\}$ che contiene totalmente (o coincide) col sistema di superficie determinato sulla $V_{3}{ }^{\left({ }^{6}\right)}$ dalle ipersuperficie cubiche dell' $S_{5}$. Poichè un'ipersuperficie eubica dell' $S_{5}$ che contenga la $V_{3}{ }^{(6)}$ si spezua nel cono quadrico $M_{4}^{(2)}$ e in un iperpiano generico $\left({ }^{28}\right)$, la dimensione $\delta_{2}$ del sistema $\{\psi\}$ è

$$
\delta_{2} \geq\left(\begin{array}{c}
5+3 \\
3
\end{array}\right)-1-6=49
$$

Il sistema di curve $\Gamma$ è contenuto totalmente nel sistema quadruplo del sistema delle curve-sezioni della $F_{q}^{\left({ }^{(6)}\right.}$. Quindi per il suo grado $n^{\prime}$ ed il suo genere $\pi^{\prime}$ si ha

$$
n^{\prime}=4^{2} \cdot 6=96, \quad \pi^{\prime}=4 \cdot 3+6 \cdot 6-3=45
$$

ciò̀ la dimensione della serie-caratteristica completa del sistema $|\Gamma|$ è $n^{\prime}-\pi^{\prime}=96-45=51$. Dunque la dimensione del sistema lineare di curve $\Gamma$ è $\delta_{1} \leq 52$. Quindi per la dimensione del sistema lineare delle $\Phi$ si ha (n. 29)

$$
\delta=\delta_{1}+\delta_{2}+1 \leq 52+49+1=102 \text {. }
$$

Poichè il sistema lineare delle ipersuperficie del quarto ordine dell' $S_{5}$ ha la dimensione $D=\left(\begin{array}{c}5+4 \\ 4\end{array}\right)-1=125$, il numero delle ipersuperficie quartiche linearmente indipendenti che contengono la $V_{3}^{(6)}$ d

$$
D-\delta \geq 125-102=23 \text {. }
$$

Di queste $\left(\begin{array}{c}5+2 \\ 2\end{array}\right)=21$ si possono considerare spezzate nel cono quadrica $M_{4}{ }^{(2)}$ ed in un'iperquadrica dell' $S_{5}$. Vi sono quindi (almeno) $23-21=2$ ipersuperficie quartiche (linearmente indipendenti) che contengono la $V_{3}{ }^{(6)}$ senza contenere il cono $M_{4}^{(2)}$. Una di esse sega dunque il cono $M_{4}^{\left({ }^{2}\right)}$ secondo la $V_{3}{ }^{(6)}$, ed ulteriormente in una quadrica $Q_{3}$.

${ }^{(2 T)}$ La $V_{3}{ }^{(6)}$ sezione completa di $M_{4}{ }^{(2)}$ con un'ipersuperficie cubica dell' $S_{5}$ è a curvesezioni di genere $\pi=4$.

(28) $\mathrm{Cfr},(27)$. 
34. Dimostriamo che esiste una conica $C_{a}$ unisecante le quadriche del fascie $|Q|$ della $V_{3}{ }^{(6)}$. Infatti, da un suo punto generico $P$ la $V_{3}^{\left({ }^{(6)}\right.}$ si proietta in una $V_{3}^{(5)}$ di un $S_{4}$. Nel successivo n. 38 dimostreremo che esiste una retta $a$ unisecante le quadriche del fascio lineare esistente nella $V_{3}{ }_{3}^{(5)}$. Un piano generico $\delta$ dell' $S_{4}$ per la retta a taglia ulteriormente la $V_{3}{ }^{(5)}$ in una quartica $C^{\left({ }^{4}\right.}$. L' $S_{3}$ che congiunge il piano $\delta$ col centro di proiezione $P$ taglia quindi la $V_{3}{ }^{(6)}$ in una carva, che si spezza in una quartica (che ha per proiezione la $C^{\left({ }^{(t)}\right)}$ e in una conica $O_{a}$ (che ha per proiezione la retta $a$ ), unisecante le quadriche del fascio $|Q|$.

35. Il vertice del cono $M_{4}^{\left({ }^{(2)}\right.}$ (n. 11) sia una retta $b$. Un $S_{4}$ generico per l' $S_{3}$ di una quadrica $Q$ della $V_{3}^{\left({ }^{(B)}\right.}$ (cioè per l' $S_{3}$ di una schiera del cono $M_{3}^{\left(z^{(}\right)}$), taglia la $V_{3}{ }^{(6)}$ ulteriormente in una superficie del quarto ordine, $F_{2}{ }^{(4)}$, a curve sezioni di genere due, e il cono $M_{4}^{(2)}$ in un $S_{3}$ dell'altra schiera (il quale è lo spazio d'appartenenza della $\left.F_{2}^{\left({ }^{(}\right)}\right)$. Quindi la quadrica $Q_{3}$, ulteriore intersezione di una $M_{4}^{(4)}$ per la $V_{3}^{\left({ }^{(6)}\right.}$ col cono $M_{4}^{(2)}$, (n. 21), si spezza in due $S_{3}$ del cono $M_{4}{ }^{(2)}$ (della schiera degli $S_{3}$ che contengono le $F_{2}{ }^{\left({ }^{(4)}\right.}$ della $V_{3}{ }^{\left({ }^{(}\right)}$). La retta $b$ risulta cosi doppia per la $V_{3}{ }^{(6)}$. Infatti, poichè $b$ è doppia per la $M_{4}{ }^{(4)}$ (n. 33), essa è quadrupla per l'intersezione completa dell' $M_{4}^{\left({ }^{(4)}\right.}$ coll' $M_{4}^{(2)}$, ed essendo doppia per la coppia di $S_{3}$, essa è doppia per la residua intersezione $V_{3}{ }^{\left({ }^{6}\right)}$.

Viceversa, una $M_{4}^{\left({ }^{(4)}\right.}$ che passi per due $S_{3}$ di un $S_{1}-$ cono $M_{4}{ }^{\left({ }^{2}\right)}$, di una medesima schiera, sega ulteriormente questo cono in una $V_{3}{ }^{\left({ }^{6}\right)}$ a curve-sezioni iperellittiche di genere $\pi=3$.

36. Supponiamo ancora che il vertice del cono $M_{4}^{(2)}$ sia una retta $b$. Consideriamo una conica $C_{a}$ unisecante le quadriche $Q$ della $V_{3}{ }^{(6)}$. Questa conica non è incidentè la retta $b$.

Infatti, se $C_{a}$ fosse incidente $b$, essa arrebbe in comune coll' $S_{3}$ di ciascuna $Q$ due punti (il punto sa $b$ e il punto d'incidenza colla relativa $Q$ ). Quindi la retta $a$, proiezione di $C_{a}$ da un suo punto su un $S_{4}$ (n. 34 ), dovrebbe appartenere agli $S_{3}$ delle proiezioni delle $Q$, mentrechè essa ha in comune cogli $S_{3}$ di quelle $Q$ un solo punto.

Introduciamo nell' $S_{5}$ un $S_{4}^{*}$ generico per la retta $b$. Indichiamo con $B_{1}$ e $B_{2}$ i suoi punti d'incontro con la conica $C_{a}$, con $\beta_{1}$ e $\beta_{2}$ i due piani che da questi punti proiettano la retta $b$, con $M_{3}^{\left({ }^{(2)}\right.}$ e $F^{\left({ }^{(}\right)}$le sezioni dell' $S_{4}{ }^{*}$ rispettivamente col cono $M_{4}^{\left({ }^{(2)}\right.}$ e la $V_{3}{ }^{(6)}$.

Una generica quadrica $Q$ ha in comune colla conica $C_{a}$ un (solo) punto $A$, ed il suo $S_{3}$ d'appartenenza taglia $l^{\prime} S_{4}^{*}$ in un piano $\beta$ del cono $M_{3}{ }^{\left({ }^{2}\right)}$. Proiettando la $Q$ da $A$ su $\beta$ si ottiene, al variare di $A$ lungo la conica $C_{a}$, una 
rappresentazione birazionale della $V_{3}^{(6)}$ sul cono quadrico $M_{3}{ }^{(2)}$ (che ha per vertice la retta $b$ ).

Il sistema lineare di superficie $|\Phi|$, rappresentativo della $V_{3}{ }^{(6)}$ sul cono $M_{3}{ }^{(2)}$, ha come elementi-base la retta $b$, la curva $C^{(\mu)}$, traccia sull' $S_{4}^{*}$ della rigata formata dalle rette delle quadriche $Q$ che passano per i punti $A$ della conica $O_{a}$.

Osserviamo inoltre che un punto generico della quadrica $Q$ passante per $B_{1}$ si proietta nel punto $B_{1}$ (mentre la conica sezione di questa $Q$ col piano $\beta_{1}$ ha come proiezione questo piano). Dunque il punto $B_{1}$ e l'analogo

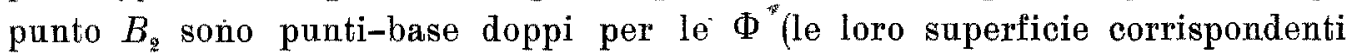
essendo quadriche).

La retta $b$ (contata assieme ai piani $\beta_{1}$ e $\beta_{2}$ ) è l'imagine della superficie $F_{z}{ }^{(0)}$, sezione della $V_{3}{ }^{\left({ }^{6}\right)}$ coll' $S_{4}$ per la $C_{a}$ e la retta $b$ (fatta eccezione della $O_{a}$ ). In particolare, ad un punto $B$ della $b$ corrisponde la $C^{(4)}$ che si trova sul cono che da. $B$ proietta $C_{a}$ (sezione di questo cono con la $M_{4}^{\left({ }^{(4)}\right.}$ che passa per due delle sue generatrici e per la conica $C_{a}$ ).

Una particolare superficie dè sistema $|\Phi|$ è la $F^{(6)}$ (sezione della $V_{3}^{\left({ }^{(6)}\right.}$ coll' $\left.S_{4}^{*}\right)$, contata assieme ai piani $\beta_{1}$ e $\beta_{2}$ (n. 30). Dunque le superficie $\Phi$ sono dell' ottavo ordine.

Alle superfioie-sezioni della $V_{3}^{\left({ }^{(6)}\right.}$ cogli $S_{4}$ per la conica $C_{a}$ corrispondono sull' $M_{3}{ }^{\left({ }^{2}\right)}$ gli $S_{4}$-coni quadrici, sezioni dell' $S_{4}-$ cono $M_{3}{ }^{(2)} \operatorname{cogli} S_{3}$ dell' $S_{4}{ }^{*}$ per i punti $B_{1}, B_{2}$; contati assieme alla superficie rigata $\Psi^{(6)}$, imagine della conica $C_{a}$ (traccia sull' $S_{4}^{*}$ dell'insieme dei piani tangenti alle $Q$ nei punti della $C_{a}$ ), avente $b$ come direttrice e $B_{1}, B_{2}$ come punti semplici.

Alle.$\infty^{1}$ superficie $E_{2}^{(4)}$ della $V_{3}^{(6)}$ corrispondono su $M_{3}^{\left({ }^{(9)}\right.}$ superficie del settimo ordine, $\Phi^{(7)}$, (che costituiscono una $\Phi^{(8)}$ assieme ad un piano generico $\beta$ del cono $M_{3}{ }^{\left({ }^{2}\right)}$ coi punti doppi $B_{1}$ e $B_{2}$; segate in coniche dai piani $\beta$ del cono $M_{3}{ }^{(2)}$. Questo fascio $\left|\Phi^{(7)}\right|$ determina su un generico piano $\beta$ un fascio di coniche, del quale due punti-base sono i punti d'incontro (esterni a $b$ ) di $\beta$ colla curva-base $C^{(\mu)}$ e gli altri due si trovano sulla retta $b$ (e sono i due punti d'incontro della $Q$, di cui $\beta$ è proiezione, colla $b$ ). Quindi elementibase del fascio $\left|\Phi^{(7)}\right|$ sono la curva $C^{(\mu)}$, la retta $b$ e due coppie di rette, rispettivamente nei piani $\beta_{1}, \beta_{2}$ e per i punti $B_{1}, B_{2}$ (proiezioni, ad esempio da $B_{1}$, dei due punti d'incontro con $b$ della $Q$ passante per $B_{1}$ ).

Proiettando infine il cono quadrico $M_{3}^{(*)}$ dal punto $B_{2}$ sopra un $S_{3}{ }^{*}$ generico per la retta $b$ e per il punto $B_{1}$. Il sistema lineare di $\Phi^{(8)}$ si proietta in un sistema lineare $\varphi^{(6)}$, con la retta quadrupla $b$ (perchè segate ${ }^{\text {in }}$ coniche dai piani per b), la curva-base $C^{(v)}$ (proiezione della $C^{(\mu)}$ ) e il puntobase doppio $B_{1}$. Al fascio di $\Phi^{(?)}$ (coi punti doppi $B_{1}$ e $B_{2}$ ) corrisponde un 
fascio di $\varphi^{(5)}$, colla retta tripla $b$, e per ulteriori elementi-base la curva $O^{(v)}$, due rette nel piano $\beta_{1}$ e due piani tangenti fissi nei punti della $b$, imagini della $b$ stessa, $\left({ }^{29}\right)$. Inoltre le $\varphi^{(5)}$ hanno lungo la $b$ un piano tangente variabile (imagine del punto d'incontro dell' $F_{a^{2}}{ }^{(4)}$ con la $C_{a}$ ).

Alla rigata $\Psi^{(6)}$ (col punto semplice $B_{2}$ ) corrisponde nell' $S_{3}^{*}$ una rigata $\Psi^{(5)}$, colla retta quadrupla $b$, il punto semplice $B_{1}$, e passante ulteriormente per la curva-base $O^{(v)}$. Le $\varphi^{(5)}$ del fascio precedentemente considerato hanno come intersezione completa con la $\Psi^{(5)}$ la retta $b$, la $C^{(v)}$ e una generatrice variabile della $\psi^{(5)}$ (imagine del punto variabile nel quale la $F_{2}^{\left({ }^{(4)}\right.}$ incontra la conica $C_{a}$ ).

In conclusione la $V_{3}{ }^{(8)}$ dell' $S_{5}$ è rappresentata nell' $S_{3}{ }^{*}$ dal sistema lineare delle $\varphi^{(6)}$ con una retta-base b quadrupla, un punto-base B doppio e una $\mathrm{O}^{\left({ }^{2}\right)}$-base, sezione d'una $\varphi^{(5)}$ con la retta tripla b e il punto doppio $\mathrm{B}$, con una $\psi^{(5)}$ con la retta quadrupla b e il punto semplice B (le quali hanno inoltre in comune una generatrice della rigata $\left.\psi^{\left({ }^{5}\right)}\right)$.

Per dare la rappresentazione analitica di questo sistema lineare di $\varphi^{(6)}$ nell' $S_{3}(x, y, z)$ affine (n. 14), scegliamo il panto $B$ nell' origine, e la generatrice comune a una $\varphi^{(5)}$ e alla $\psi^{(5)}$ nella retta impropria del piano $(x z)$. Si ottiene allora

$$
\begin{gathered}
{\left[\bar{F}_{3} x+\bar{G}_{4} y+\bar{H}_{3} z\right]\left(\lambda_{0} x+\lambda_{1} y+\lambda_{2} z\right)+} \\
+\left[\bar{F}_{2}^{2} x^{2}+\bar{L}_{3} x y+\bar{M}_{3} y^{2}+\left(\bar{G}_{2} x+\bar{N}_{3} y+\bar{H}_{2} z\right) z\right] F_{1}+\lambda_{5} \Phi_{6}=0
\end{gathered}
$$

dove $\Phi_{6}=0$ rappresenta una $\varphi^{(5)}$ con la $b$ quartupla, il $B$ doppio, passante per la $C^{(4)}$, senza passare per la retta impropria del piano $(x z)$.

37. Il vertice del cono $M_{4}^{(2)}$ dell' $S_{5}$ sia ora un piano $\alpha$ (n. 11). Quindi il cono contiene un solo sistema di spazi lineari $S_{3}$. Poichè la $V_{3}{ }^{(6)}$ è la sezione residua dell' $M_{4}^{\left({ }^{(2)}\right.}$ con una $M_{4}{ }^{(4)}$ che passa per due di questi $S_{3}$ (n. 33), il piano $\alpha$ è doppio per la $M_{4}{ }^{(4)}$ è, di conseguenza, anche per la $V_{3}{ }^{(6)}$.

Indichiamo con $O_{a}$ una conica unisecante le quadriche $Q$ della $V_{3}{ }^{(5)}$ (n. 34). Consideriamo nell' $S_{5}$ della $V_{3}{ }^{|6\rangle}$ uno spazio generico $S_{4}^{*}$. Indichiamo con $b$ la sua retta intersezione col piano $x$, con $B_{3}$ e $B_{2}$ i suoi punti d'incontro colla conica $O_{a}$, con $M_{3}{ }^{(2)}$ il cono quadrico (che ha per vertice la

${ }^{(29)}$ Abbiamo visto che ad un punto $B$ della $b$ (come retta dell' $M_{3}^{(2)}$ ) corrisponde una $C^{(4)}$ della $V_{3}^{(6)}$, che ha doppio il punto $B$ della $b$ (come retta della $V_{3}^{(6)}$ ). Profettando il cono $M_{3}{ }^{(2)}$ nell' $S_{3}{ }^{*}$, al punto $B$ della $b$ (come retta dell' $S_{3}{ }^{*}$ ) corrisponde ancora la $C^{(4)}$ della $V_{3}{ }^{(6)}$, che passa due volte per il $B$ della $V_{3}^{(6)}$ in corrispondenza a due particolari piani per la $b$ dell' $S_{3}{ }^{*}$. 
retta b) traccia dell' $M_{4}^{(2)}$, con $F^{(6)}$ la superficie-sezione colla $V_{3}^{(6)}$. Siano $\beta_{1}$ e $\beta_{2}$ i piani che proiettano la retta $b$ rispettivamente da $B_{1}$ e $B_{2}$.

Una quadrica generica $Q$ della $V_{3}^{\left({ }^{(6)}\right.}$ ha in comune colla conica $C_{a}$ un punto $A$, ed il suo $S_{3}$ d'appartenenza sega $l^{\prime} S_{4}{ }^{*}$ in un piano $\beta$ del cono $M_{3}{ }^{[2]}$. Proiettando la $Q$ da $A$ su $\beta$ si ottiene, al variare della $Q$, una rappresentazione birazionale della $V_{3}^{\langle 6\rangle}$ sul cono $M_{3}^{\langle 2\rangle}$.

Il sistema lineare di superficie $|\Phi|$, rappresentativo della $V_{3}{ }^{\langle 6\rangle}$ sul cono $M_{3}{ }^{(2)}$, ha come elementi-base la retta $b$, la curva $C^{\langle\mu\rangle}$ traccia sull' $S_{4}{ }^{*}$ della rigata formata dalle rette delle $Q$ che passano per i punti $A$ della conica $C_{a}$, ed i punti doppi $B_{1}$ e $B_{2}$ (n. 28). Una particolare superficie del sistema $|\Phi|$ è la $F^{(6)}$, contata assieme ai piani $\beta_{1}$ e $\beta_{z}$. Dunque le $\Phi$ sono dell' ottavo ordine.

Alle superficie-sezioni della $V_{3}^{(5)}$ cogli $S_{4}$ per la conica $C_{a}$ corrispondono sull $M_{3}{ }^{(2)}$ i coni quadriei, sezioni $\operatorname{cogli} S_{3}$ per i punti $B_{1}$ e $B_{2}$, contati assieme alla superficie rigata $\psi^{(b)}$, imagine della conica $C_{a}$, avente $b$ come direttrice (traccia nell' $S_{4}^{*}$ della varietà dei piani tangenti alle $Q$ nei punti della $\mathrm{C}_{a}$ ). Questa rigata e la $F^{(6)}$ sono tangenti nei punti $B_{1} \in B_{2}$ (n. 28).

Al piano $\alpha$ corrisponde sull' $M_{3}{ }^{2}$ una superficie $\Phi^{(6)}$ (che assieme a due piani $\beta$ del cono $M_{3}{ }^{(2)}$ costituisce una superficie del sistema $\left|\Phi^{(8)}\right|$, coi punti doppi $B_{1}$ e $B_{2}$. Un generico piano $\beta$ sega rispettivamente $F^{\{6\rangle}$ e $\Phi^{(6)}$ in coniche che hanno gli stessi due punti in comune colla retta $b$, cioè $\mathbf{i}$ due punti comuni alla retta $b$ e alla quadrica $Q$ che ha per imagine il piano $\beta$. Questa $\Phi^{(6)}$ contiene (come la $F^{(6)}$ e la $\Psi^{(6)}$ ) la curva-base $C^{(\mu)}$, anzi la $C^{\langle\mu\rangle}$ $\grave{e}$, assieme alla retta $b$, l'intersezione completa della $\Psi^{(6)}$ colla $\Phi^{(a)}$.

Proiettiamo il cono $M_{3}^{(2)}$ dal punto $B_{1}$ sopra un $S_{3}^{*}$ dell' $S_{4}^{*}$, generico per la retta $b$. Il sistema lineare di superficie $\Phi^{(8)}\left(\mathrm{col}\right.$ punto doppio $\left.B_{1}\right)$ si proietta in un sistema, lineare di superficie $\varphi^{(6)}$, colla retta quadrupla $b$ (perchè segate in coniche dai piani per $b$ ), la curva-base $C^{(v)}$ (proiezione della $C^{\left(\mu^{\prime}\right)}$ e il punto doppio $B$ (proiezione di $B_{2}$ ). La $\Psi^{(6)}$ (coi punti semplici $B_{1}$ e $\left.B_{2}\right)$ si proietta in un $\psi^{(5)}$, colla retta quadrupla $b$ e il punto semplice $B$. La $\Phi^{(6)}$ (coi punti doppi $B_{1}$ e $B_{2}$ ) si proietta in una $\varphi^{(4)}$ colla retta doppia $b$ ed il punto doppio $B$.

In conclusione la nostra $V_{3}{ }^{(6)}$ è rappresentata nell' $S_{3}{ }^{*}$ dal sistema lineare delle p $^{|6\rangle}$ con una retta quadrupla $\mathrm{b}$, un punto doppio $\mathrm{B}$ e una $\mathrm{O}^{12}-b a s e$, sezione d'una $\psi^{(5)}$, colla retta quadrupla b e il punto semplice $\mathrm{B}$, con una $\mathrm{p}^{(4)}$, colla retta doppia b e il punto doppio B.

Per ottenere di questo sistema lineare di $\varphi^{(6)}$ la rappresentazione analitica. nell' $S_{3}(x, y, z)$ affine (n. 14), scegliamo il punto $B$ nell'origine. Si trova 
allora direttamente

$$
\begin{aligned}
& {\left[\bar{F}_{2} x^{2}+\bar{G}_{2} x y+H_{2} y^{2}+\left(\bar{L}_{2} x+\bar{M}_{2} y+\bar{N}_{2} z\right) z\right] F_{2}+} \\
& \quad+\left[\bar{F}_{4} x+\bar{G}_{4} y+\bar{H}_{4} z\right]\left(\lambda_{3} x+\lambda_{4} y+\lambda_{5} z\right)=0 .
\end{aligned}
$$

8 6. La $V_{3}^{\left({ }^{(5)}\right.}$ dell' $S_{4}$ a curve-sezioni iperellittiche di genere $\pi=3$.

35. La $V_{3}^{(5)}$ non sia un cono. Le quintiche sezioni piane della $V_{3}^{(5)}$ sono iperellittiche e di genere $\pi=3$, quindi hanino un punto triplo; cioè la $V_{3}{ }^{\langle 5\rangle}$ ha un piano triplo $\alpha$. Le quadriche del fascio $|Q|$ sono segate sulla $V_{3}{ }^{(5\rangle}$ dagli $S_{3}$ per il piano $\alpha$.

Dimostriamo che la $V_{3}{ }^{|5\rangle}$ contiene delle rette unisecanti le quadriche del fascio $Q$. Perciò consideriamo 6 quadriche generiche $Q^{i}(i=1, \ldots, 6)$. Da un punto generico $P$ della quadrica $Q_{1}$ le quadriche $Q_{2}$ e $Q_{3}$ si proiettano, dentro allo spazio lineare $S_{3}$ della quadrica $Q_{4}$ in due quadriche $Q_{2}{ }^{\prime}$ e $Q_{3}{ }^{\prime}$ le quali hanno in comune con la quadrica $Q_{4}$ (in generale) otto punti. Cioè dal punto $P$ della $Q_{1}$ escono otto rette unisecanti le altre tre quadriche. Una di queste rette sarà contenuta nell' $S_{3}$ della quadrica $Q_{1}$ unicamente se le altre tre quadriche hanno in comune un punto del piano $\alpha$. Questo punto è allora comune a tutte le quadriche del fascio $|Q|$, cioè il punto è quadruplo (n. 40) per la $V_{4}{ }^{\langle 3\rangle}$. Dunque anche nel caso più sfavorevole che la $V_{3}^{(4)}$ abbia nel piano $\alpha$ una conica luogo di punti tripli, esistono ancora due rette che escono da $P$ e sono incidenti le tre quadriche $Q_{2}, Q_{3}, Q_{4}$, senza essere incidenti il piano $\alpha$.

Al variare del punto $P$ lungo la quadrica $Q_{1}$, le rette che passano per $P$ $\Theta$ sono incidenti alle altre tre quadriche, senza esserlo al piano $\alpha$, generano una varietà algebrica $M_{3}$. Questa $M_{3}$ è segata dall' $S_{3}$ della quadrtea $Q_{5}$ in una superficie, che ha in comune colla $Q_{5}$ una curva. Le $\infty^{1}$ rette generatrici dell' $M_{3}$ che passano per questa curva sono incidenti le 5 quadriche $Q_{1}, \ldots, Q_{5}$. La rigata generata da queste $\infty^{1}$ generatrici è segata dall' $S_{3}$ della quadrica $Q_{6}$ in una curva, la quale ha in comune con la $Q_{6}$ alcuni punti. La generatrice passante per uno di questi punti si appoggia alle sei quadriche $Q_{1}, \ldots, Q_{b}$, ciò̀ appartiene alla $V_{3}^{(5)}$ (ed è unisecante le quadriche del fascio $|Q|$ ), c. v. d.

39. Sia $a$ una delle rette unisecanti le quadriche del fascio $|Q|$. Fissiamo nell' $S_{4}$ della $V_{3}^{\left|{ }^{5}\right\rangle}$ uno spazio $S_{3}^{*}$ generico. Indichiamo con $b$ la sua retta d'intersezione col piano $\alpha$ e ron $B$ il suo punto $d$ 'incontro colla retta $a$.

Una quadrica generica $Q$ del fascio $\mid Q$, ha in comune colla retta $a$ un punto $A$, ed il suo spazio d'appartenenza sega $l^{\prime} S_{3}^{*}$ in un piano $\beta$ del fascio di asse $b$. Proiettiamo la quadrica $Q$ da $A$ sul piano $\beta$. Al variare della $Q$ otteniamo cosi una rappresentazione birazionale della $V_{3}^{\left\langle{ }^{|j\rangle}\right.}$ sull' $S_{3}^{*}$. 
Ricerchiamo il sistema lineare di superficie $|\varphi|$ corrispondente in questa rappresentazione al sistema delle superficie-sezioni della $V_{3}^{\langle\text {(5) }}$. Gli elementibase del sistema $|\varphi|$ saranno la retta $b$, il punto $B$ e la curva $C^{\langle\nu\rangle}$ traccia sull' $S_{3}{ }^{*}$ della rigata formata dalle rette delle quadriche $Q$ che si appoggiano ai punti $A$ della retta $a$.

Si osservi che un punto generico della quadrica $Q$ passante per $B$ si proietta nel punto $B$ (mentre la conica sezione di questa $Q$ col piano $\beta_{1}$ per $b$ e $B$ ha come proiezione questo piano). Dunque il punto $B$ è un puntobase (almeno) doppio per le $p$ (la sua superficie corrispondente essendo una quadrica).

Una particolare superficie del sistema $|\varphi|$ è la superficie $F^{(5)}$ sezione

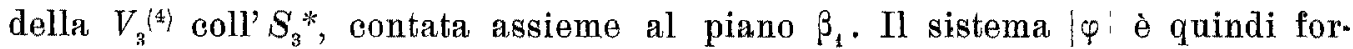
mato da superficie del sesto ordine, $\varphi^{(6)}$, con la retta quadrupla $b$ e il punto doppio $B$. La curva-base $O^{(\nu)}$ appartiene alla $F^{(5)}$.

La curva $C\langle v\rangle$ appartiene anche alla rigata $\psi$, colla direttrice $b$ sezione dell' $S_{3}^{*}$ coi piani tangenti alle quadriche $Q$ nei punti $A$ della retta $a$.

Alla superficie-sezione $F_{2}{ }^{(5\rangle}$ della $V_{3}{ }^{(5)}$ con un $S_{3}$ generico per lá retta $a$ corrisponde nell' $S_{3}{ }^{*}$ il piano $\gamma$ per il punto $B$ sezione dell' $S_{3}$ coll' $S_{3}{ }^{*}$, contato assieme alla rigata $\psi$, immagine della retta $a$. La $\psi$ è quindi una superficie del quinto ordine, $\psi^{(5)}$, colla retta quadrupla $b$. Sul piano $\gamma$ la $F_{2}^{(5)}$ è rappresentata dal sistema lineare delle sestiche con un punto quadruplo (sezione di $\gamma$ con b), il punto doppio $B$ e 11 punti-base semplici (11 affinchè íl grado del sistema sià 5), sezioni del piano $\gamma$ colla curva $C^{(y)}$. Poichè (come ora proveremo) $B$ è doppio per la $C^{(\nu)}$, sarà $y=13$.

La curva $C^{(13)} \dot{e}$, assieme alla retta $b$, l'intersezione completa delle superficie $F^{(5)}$ e $\psi^{(5)}$. Queste due superficie sono tangenti nel punto $B$; cio che prova che $B$ è un punto doppio della $C^{\{13}$. Infatti la varietà a tre dimensioni generata dai piani tangenti alla $Q$ nei punti della retta $a$ e la $V_{3}^{\mid{ }^{|5\rangle}}$ hanno in un punto generioo $A$ della retta $a$ (in particolare in $B$ ) lo stesso $S_{3}$-tangente, individuato dalla retta $a$ e dalle due rette della $Q$ che escono da $A$. Quindi le superficie $\psi^{(6)}$ e $F^{(5)}$, che sono rispettive sezioni di quelle varietà coll' $S_{3}^{*}$, avranno in $B$ lo stesso piano tangente, (n. 28 ).

Le superficie del fascio definito nell' $S_{3}^{*}$ dalle $F^{(5)}$ e $\psi^{(5)}$ sono lassieme al piano $\beta_{1}$ ) le imagini delle superficie-sezioni della $V_{3}{ }^{\left\langle{ }^{5}\right\rangle} \operatorname{cogli} S_{3}$ dell' $S_{4}$ per il piano $\beta_{1}$. In questo fascio di superficie del quinto ordine (colla retta tripla $b$ ), tangenti nel punto $B$, vi è una col punto doppio $B$. Essa è l'imagine del piano $\alpha$. Infatti alle sezioni della $V_{3}^{\{5\rangle} \operatorname{cogli} S_{3}$ per $\alpha$ corrispondono i piani per la retta $b$ e una superficie $\varphi^{(5)}$, imagine del piano $\alpha$. La sezione della $\varphi^{(5)}$ con un piano $\beta$ per la $b$ è (oltre la retta $b$ ) la conica proiezione della conica 
del piano $\alpha$, sezione con $\alpha$ della quadrica $Q$ dell' $S_{3}$ per $\alpha$ individuato dal piano $\beta$, fatta dal rispettivo punto $A$ della retta $a$. Il punto $B$ è doppio per la $\varphi^{(5)}$, poichè esso corrisponde àd una conica (del piano $\propto$ ). La curva $C^{(13)}$ è dunque, assieme alla retta $b$, anche l'intersezione completa delle super. ficie $\psi^{(5)}$ e $\varphi^{(5)}$. La $C^{(13)}$ e anche sezione delle superficie $\varphi^{(5)}$ e $F^{(5)}$, di oui fa parte la retta tripa $b$ e tre rette infinitamente vicine alla $b$. Questa ultima circostanza si verifica osservando che le coniche, sezioni delle $\varphi^{(5)}$ e $F^{(5)}$ con un piano $\beta$ generico per la retta $b$ hanno in comnne due punti della retta $b$ (cioè i punti d'incontro della $b$ colla quadrica $Q$ relativa al piano $\beta$ ).

In conclusione, il sistema lineare rappresentativo nell' $S_{3}{ }^{*}$ della $V_{3}^{(5)}$ è dato dalle superficie $\phi^{(0)}$, del sesto ordine, con una retta-base b quadrupla ed una curva-base $\mathrm{C}^{(13)}$, sezione di due superficie del quinto ordine colla retta $\mathrm{b}$ rispettivamente tripla e quadrupla, tangenti in un punto generico $\mathrm{B}$, il quale è doppio per le $\varphi^{\langle 6\rangle}$.

Per ottenere di questo sistema di $\varphi^{(6)}$ la rappresentazione analitica nell' $S_{3}(x, y, z)$ affine (n. 14) scegliamo l'origine nel punto $B$. Si ottiene allora direttamente

$$
\begin{gathered}
\left(\bar{F}_{4} x+\bar{G}_{4} y+\bar{H}_{4} z\right)\left(\lambda_{0} x+\lambda_{1} y+\lambda_{2} z\right)+ \\
+\left[\bar{L}_{4} x^{2}+\bar{M}_{4} x y+\bar{N}_{4} z+\left(\bar{O}_{4} x+\bar{P}_{4} y+\bar{Q}_{4} z\right) z\right] F_{1}=0
\end{gathered}
$$

40. Il sistema lineare di $\varphi^{\langle 6\rangle}$ ora considerato ammette moltissimi casi particolari, a seconda delle particolarità ché può presentare la $C^{13}$-base. Può anche darsi che, in base a queste particolarità, si possa, con una trasformazione cremoniana, abbassare l'ordine del sistema lineare di superficie.

Ad esempio, se una retta $s$ per il punto-base doppio $B$ è ulteriormente quadrisecante la $C^{\{13\rangle}$, essa è una retta-fondamentale di $\left|\varphi^{(6)}\right|$, imagine di un punto quadruplo $A$ della $V_{3}^{(5)}$. Il sistema omaloidico di superficie del $6^{\circ}$ ordine dell' $S_{3}^{*}$ formato dalle superficie del sistema $\left|\varphi^{(6)}\right|$ che hanno come ulteriore elemento-base la retta $s$, trasforma il sistema $\left|\varphi^{(b)}\right|$ nel sistema delle $\varphi^{(5)}$, con una retta-base tripla $b$ e una $C$-base. sezione di una $\varphi^{(5)}$ con una $\varphi^{(4)}$, colla retta tripla $b$.

Questa considerazione finale può essere estesa a diversi dei sistemi lineari precedentemente considerati. 